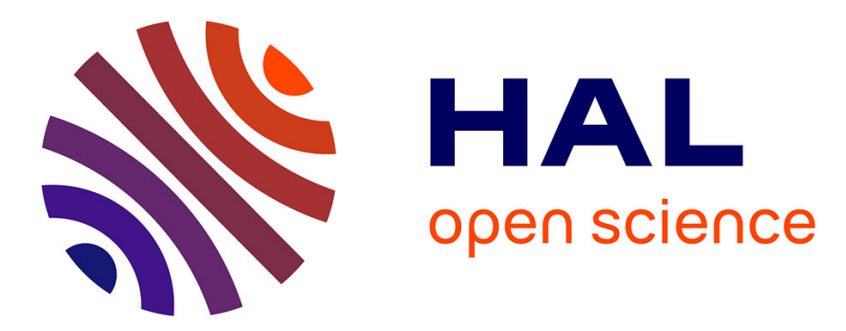

\title{
Analysis and approximation of one-dimensional scalar conservation laws with general point constraints on the flux
}

Boris Andreianov, Carlotta D Donadello, Ulrich Razafison, Massimiliano D

Rosini

\section{To cite this version:}

Boris Andreianov, Carlotta D Donadello, Ulrich Razafison, Massimiliano D Rosini. Analysis and approximation of one-dimensional scalar conservation laws with general point constraints on the flux. 2016. hal-01418272v2

\section{HAL Id: hal-01418272 \\ https://hal.science/hal-01418272v2}

Preprint submitted on 13 Jul 2017

HAL is a multi-disciplinary open access archive for the deposit and dissemination of scientific research documents, whether they are published or not. The documents may come from teaching and research institutions in France or abroad, or from public or private research centers.
L'archive ouverte pluridisciplinaire HAL, est destinée au dépôt et à la diffusion de documents scientifiques de niveau recherche, publiés ou non, émanant des établissements d'enseignement et de recherche français ou étrangers, des laboratoires publics ou privés. 


\title{
ANALYSIS AND APPROXIMATION OF ONE-DIMENSIONAL SCALAR CONSERVATION LAWS WITH GENERAL POINT CONSTRAINTS ON THE FLUX
}

\author{
BORIS ANDREIANOV, CARLOTTA DONADELLO, ULRICH RAZAFISON, AND MASSIMILIANO D. ROSINI
}

\begin{abstract}
We introduce and analyze a class of models with nonlocal point constraints for traffic flow through bottlenecks, such as exit doors in the context of pedestrians traffic and toll gates in vehicular traffic. Constraints are defined based on data collected from non-local in space and/or in time observations of the flow. We propose a theoretical analysis and discretization framework that permits to include different data acquisition strategies; a numerical comparison is provided. Nonlocal constraint allows to model, e.g., the irrational behavior ("panic") near the exit observed in dense crowds and the capacity drop at tollbooth in vehicular traffic.

Existence and uniqueness of solutions are shown under suitable and "easy to check" assumptions on the constraint operator. A numerical scheme for the problem, based on finite volume methods, is designed, its convergence is proved and its validation is done with an explicit solution. Numerical examples show that nonlocally constrained models are able to reproduce important features in traffic flow such as self-organization.
\end{abstract}

Keywords: scalar conservation law, nonlocal point constraint, well-posedness, fixed point argument, finite volume scheme, convergence of approximations, vehicular traffics, crowd dynamics

2010 AMS Subject classification: 35L65, 90B20, 65M12, 76M12

\section{CONTENTS}

1. Introduction

1.1. Well posedness and approximation of conservation laws with point constraints on the flow

1.2. State of the art in modelling road and pedestrian traffic with point constraints

1.3. The general framework of the present paper

1.4. Need for new advanced models

1.5. Structure of the paper

2. Assumptions and definitions

2.1. Assumptions

2.2. Entropy solutions for models with point constraints

3. Existence and uniqueness of entropy solutions to constrained problems

3.1. Evolution operators

3.2. A well-posedness result

3.3. Existence results

4. Basic examples of constraint operators

5. Finite volume approximation of nonlocally constrained problems

5.1. Non-local constrained finite volume method

5.2. Convergence of the scheme

6. Examples of discrete constraint operators

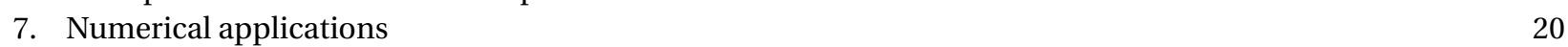

$\begin{array}{ll}\text { 7.1. Validation of the numerical scheme } & 20\end{array}$

7.2. Numerical simulations 23

\begin{tabular}{lr}
\hline 8. Conclusions & 24 \\
\hline Acknowledgnent & 23
\end{tabular}

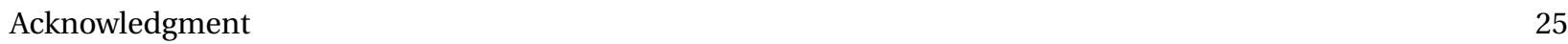

\begin{tabular}{ll}
\hline References & 25
\end{tabular}

\section{INTRODUCTION}

1.1. Well posedness and approximation of conservation laws with point constraints on the flow. In this paper we study Cauchy problems for one-dimensional scalar conservation laws subject to point constraints of the form
(1.1a)
$\rho_{t}+f(\rho)_{x}=0$,
$x \in \mathbb{R}, t \in(0, T]$,
(1.1b)
$f(\rho)\left(t, 0^{ \pm}\right) \leq q(t)$,
$t \in(0, T]$,

Date: July 10, 2017. 
Above $\rho$ is the unknown variable, $f$ is the flux, $q$ gives the maximal flow allowed through $x=0$ and $\rho_{0}$ is the initial datum. Such problems naturally arise in modelling the flows of conserved quantities through bottlenecks, namely at points where an obstacle hinders the flow. At last, the final time $T>0$ will be called time horizon in the sequel.

Despite the concept of point constraints is stated in a general mathematical framework, see for instance [48], according to the authors' knowledge, problem (1.1) is so far applied only in two frameworks: crowd dynamics 3 [2 4, 18, 19, 20, 22, 24, 25] and vehicular traffics [8, 5, 6, 16, 21, 23, 28, 29, 30, 32]. However, it is easy to envisage the application of (1.1) in other fields of research, such as biology (e.g., to model flows of biological substances across cell membranes), biomedicine (e.g., to model blood flows in vessels through thromboses), internet traffic engineering (e.g., to model flows of data through routers or proxies), etc. Moreover, recent empirical data for both pedestrian and vehicular flows highlight phenomena such as capacity drop, self-organization, Braess' paradox, etc., that can not be reproduced by the macroscopic models already available. However, modifications can be introduced in the basic models in order to capture such specific features of real traffic flows.

In the literature the existence results for models based on (1.1) are achieved by means of mathematical tools developed ad hoc for each model. As a consequence, an existence result obtained for a model can be hardly adapted to other models, even if they are slight modifications of the original one. Indeed, for instance, the existence results in [5, 4, 19, 21, 28, 30] are obtained by applying the wave-front tracking method proposed by Dafermos in [26] and improved later on by Di Perna [31] and Bressan [13], see [14 37] and the references therein for more details. However, this method can be hardly adapted from one model to another one because it relies on a case-by-case study of all the possible interactions, that strictly depend on the model under consideration.

The lack of general hypotheses that guarantee the existence of entropy solutions for models based on 1.1 is a serious obstacle toward a wider application of (1.1). For instance, the examples proposed in Section 1.4 and the corresponding numerical simulations given in Section 7 consist of models which are extremely close to the one proposed in [4], are of some interest by them own and, at the same time, seem excessively expensive to treat with the wave-front tracking machinery.

We deal in this paper with modifications to [1.1a that are quite practical because they do not modify the structure of the fundamental model nor of its discrete counterparts, but they add complexity due to the introduction of a coupling with operators prescribing point constraints $1.1 \mathrm{~b}$ non-locally depending on the flow. Simulations based on elementary splitting numerical procedures for resolving the coupling between the flow and the non-local constraint permit testing and adaptation of models at low cost. Our aim is to provide a mathematically rigorous set of general and "easy to check" hypotheses that guarantee existence (and sometimes uniqueness) of entropy solutions to [1.1a) coupled to wide classes of constraint operators described in Section 2 We highlight the results presented in Theorem 3.1. Corollary 3.1 and Theorem 3.2 in Section3 In Section 4 we give examples of problems of the form (1.1) that can be used as building blocks to construct more complex problems. As our well-posedness results in this paper rely on fixed point techniques, they are not constructive or they do not lead to practical algorithms for construction of solutions. To palliate this drawback we also introduce a general numerical scheme and "easy to check" hypotheses that guarantee the convergence of the scheme to entropy solutions to (1.1), see Section 5 . Moreover we apply in Sections 6 and 7 the numerical scheme to the building blocks introduced in Section 4

1.2. State of the art in modelling road and pedestrian traffic with point constraints. Point constraints were introduced into the basic LWR model in order to account for sharply localized in space phenomena that may occur at exits, traffic lights, toll gates, acting as obstacles for the traffic flow prescribed by LWR.

1.2.1. Applications to crowd dynamics. In [24] the authors apply [1.1 to model pedestrians evacuating a corridor through an exit door located in $x=0$. In this framework, $\rho \in\left[0, \rho_{\max }\right]$ is the density of pedestrians and $f:\left[0, \rho_{\max }\right] \rightarrow$ $\left[0, f_{\max }\right]$ the flux of pedestrians. In order to reproduce the capacity drop of the door in presence of high densities, they consider the following basic expression for the constraint

$$
q(t)= \begin{cases}q_{L} & \text { if } \rho\left(t, 0^{-}\right)<R, \\ q_{H} & \text { if } \rho\left(t, 0^{-}\right)>R,\end{cases}
$$

where $q_{L}$ and $q_{H}$ are, respectively, the efficiency of the door at low and high densities, $0<q_{H}<q_{L}$, and $R>0$ is the threshold between low and high densities. Let us recall that the main assumptions of the model are empirically confirmed in [36]. The existence of entropy solutions is proved in [25. 47] by applying the wave-front tracking method. Numerical approximations of the model are developed in [18, 19]. We underline that the model is able to reproduce the Braess' paradox for pedestrian flows [38, as the examples given in [20, 22, 25] show.

According to the above expression of the constraint $q$, the capacity drop of the door has a non-realistic behaviour since it is instantaneous when a high density reaches the door and it only depends on the density at the door. More realistic models should reproduce a gradual decay in the efficiency of the door as the pedestrians accumulate close to it, see [17, Figure 1]. Moreover, according to the above model, during the evacuation of pedestrians that are initially uniformly distributed, once the efficiency of the door falls, it remains constant until the very last pedestrian is evacuated, see [24, Figure 9]. On the contrary, in real life the efficiency of the door gradually increases as the number of the 
remaining pedestrians to be evacuated becomes smaller and smaller, see again [17, Figure 1]. All these considerations lead the authors of the recent paper [4] to the following expression for the constraint

$$
q(t)=p(\xi(t)) \quad \text { with } \quad \xi(t)=\int_{-\infty}^{0} w(x) \rho(t, x) \mathrm{d} x,
$$

where $w$ is a weight supported in some upstream bounded neighbourhood of $x=0$ and $p$ is a given non-increasing map that encodes the influence on the efficiency of the door of some subjective crowd density near the door. In fact, the quantity $\xi$ has the meaning of the crowd density as perceived by a pedestrian located just before the door. The Riemann problems corresponding to [1.1], 1.2] are studied in detail in [2], where it is also investigated how the regularity of $p$ impacts the well-posedness of the problem, in particular in the case of a piecewise constant function $p$, which is the relevant one for the numerical applications. The numerical approximation of its solutions together with its qualitative validation are proposed in [3], where the convergence of the numerical scheme is proved by reduction to the case where $q$ is a given function of time. It is shown in [3] that the model is able to reproduce the main effects related to the capacity drop, that are the faster is slower effect [35] and the Braess' paradox for pedestrian flows [38].

Well-posedness of [1.1], [1.2] is proved in [4], under the Lipschitz continuity assumption on the function $p$. This work is based on a wave-front tracking method with discretized (piecewise constant) function $p$. This construction has some drawbacks (actually we have a sequence of ill-posed problems converging to a well-posed one), we refer to [2] for the details. Therefore, even if the wave-front tracking strategy proved useful in the theoretical analysis, in [3] we prefer to use a simple finite volume scheme for illustrating some key features of pedestrian flows in the context of model [1.1, [1.2].

1.2.2. Applications to vehicular traffics. Colombo and Goatin apply in [21] the concept of point constraints to the first order model for road traffic by Lighthill, Whitham [42] and Richards [46], with the goal to reproduce the effects of obstacles along the road, like toll gates, traffic lights or localized construction sites. The resulting model reads [1.1], where $q$ is a function of time given beforehand, $\rho \in\left[0, \rho_{\max }\right]$ is the density of vehicles and $f:\left[0, \rho_{\max }\right] \rightarrow\left[0, f_{\max }\right]$ is the flux function that satisfies the following assumption

(F) $f$ belongs to $\operatorname{Lip}\left(\left[0, \rho_{\max }\right] ;\left[0, f_{\max }\right]\right)$ and is bell-shaped, that is $f(0)=0=f\left(\rho_{\max }\right)$

and there exists $\rho_{c} \in\left(0, \rho_{\max }\right)$ such that $f^{\prime}(\rho)\left(\rho_{c}-\rho\right)>0$ for a.e. $\rho \in\left[0, \rho_{\max }\right]$.

The existence of a unique solution is proved in [21] by using the wave-front tracking method. One of the main difficulties in this approach is the proof of a uniform bound for the total variation of the solution. The authors show that $t \mapsto \operatorname{TV}(\rho(t))$ may increase; hence, to overcome this difficulty they rather prove that $t \mapsto \operatorname{TV}(\psi(\rho(t)))$ does not increase, where $\psi(\rho)=\int_{\rho_{c}}^{\rho}\left|f^{\prime}(r)\right| \mathrm{d} r$. Only recently in [16] an estimate for the local total variation of the solution is provided. The interpretation of the model of [21] in terms of the general theory of discontinuous-flux conservation laws ([7]) and convergence of numerical approximations by means of a monotone finite volume scheme are addressed in [6]. Use of more general fluxes within the same model is the subject of [19], where a sharper definition of entropy solution is also proposed. Note that the model of [21] can be applied in traffic management, e.g. to synchronize traffic lights, see [23], see also the numerical simulations in [6].

Finally, for the sake of completeness, let us also briefly recall three recent generalizations of (1.1), even if the results of the present paper do not directly apply to them. The first one is the application of the theory of point constraint to the second order model for road traffic by Aw, Rascle [9] and Zhang [52], see [8] 5, 32, 33]. Clearly, the resulting model does not fit in the framework of the present paper, which deals with scalar conservation laws. However we will give comments and remarks related also to this model. The second generalization that we want to recall is that one proposed in [28 29, 30], where the authors introduce the concept of moving point constraint to model the influence of a slow and large vehicle on road traffic, problem that is considered also in [12, 40] but with different approaches. Finally, in [11,27] the authors apply the theory of point constraint to phase transition models.

1.3. The general framework of the present paper. In [21] and in [24], $q$ is given beforehand as a function of time and as a function of the density at $x=0^{-}$, respectively. Here, we consider a problem [1.1] subject to the more general class of point constraints

$$
q(t)=\mathscr{Q}[\rho](t) \text { for a.e. } t \in[0, T],
$$

where

$$
\mathscr{Q}: \mathbf{C}^{\mathbf{0}}\left([0, T] ; \mathbf{L}^{\mathbf{1}}(\mathbb{R} ; \mathbb{R})\right) \longrightarrow \mathbf{L}^{\mathbf{1}}\left([0, T] ;\left[0, f_{\max }\right]\right) .
$$

As a result, the operator $\mathscr{Q}$ may be non-local both in time and space, and it may account for psychological features that strongly influence the dynamics of a living system. Additional structure restrictions on $\mathscr{Q}$ will be imposed later, see Section 2.1 Moreover, to shorten notation and calculations, in the sequel we will assume that $\mathscr{Q}$ does not depend explicitly on time; the general case does not present additional analytic difficulties.

The basic example of such constraint operator is [1.2], analyzed in [4]. Let us recall that the wave-front tracking approach used in [4] provides well posedness results and allows for a rather precise study of the profiles of the solutions. Unfortunately, it requires an extremely careful case by case study of all possible interactions and the intermediate results obtained for the specific model cannot be adapted in a straightforward way to different models, even very close 
to the original model of [4]. From the general viewpoint, once the study of a specific model is advanced, one would like to consider other similar models, to compare them and to check which one fits the best with real life observations (see in particular Section 7). This paper provides some abstract general tools to address existence and uniqueness for LWR-based models with point constraints. Heading towards applications, we put forward a generic numerical scheme, whose convergence is proved under suitable hypotheses. To this end, we exploit and extend the approach of [3], both at the theoretical and at the numerical level. We describe a class of non-locally constrained models for which the existence, the approximation results and (under stronger assumptions) the uniqueness of solutions can be derived. Namely, starting from the well-posedness results and established approximation techniques for (1.1) (see [6, 21]), using fixed point arguments we are able to address solvability of non-locally constrained problems of the kind (1.1), (1.3). Further, as in [3] we combine the simple finite volume strategy of the work [6] for numerical computation of the solution $\rho$ to 1.1 with the fractional step technique, taking into account the evolution of the constraint $q=\mathscr{Q}[\rho]$. For compact operators $\mathscr{Q}$, we justify convergence of the resulting algorithm. Finally we use the scheme to simulate crowd or vehicle motions for some model cases, where non-local constraints (with space and/or time non-locality) may be of interest.

1.4. Need for new advanced models. In this section, we focus on modelling considerations that are the main source of inspiration for the paper; we also prepare the ground for the numerical illustrations of Section 7 . Readers mainly interested in theoretical aspects may skip this section; concise examples illustrating the application of the theoretical results can be found in Section 4 .

Introduction of non-locality into the LWR model with point constraint of [21] permitted to reproduce different aspects of non-monotone behavior related to capacity drop, such as Braess' paradox and Faster Is Slower feature (see [3]). However the ADR model, as developed in [4] and tested in [3] in the context of pedestrian flows upstream narrow exits, presents several drawbacks. They are related to oscillations of density near the exit, to the too simplified dynamics of the subjective density variable $\xi$ governing the constraint level $q$, and to the inability of ADR to reproduce self-organization features observed in dense (but not too dense) pedestrian flows. Moreover, ADR is not appropriate for typical situations that arise in road traffic management because it is based on instantaneous data acquisition in a small vicinity of the exit; while management of, e.g., toll gates and traffic lights should be based on data taken at distant sites and with time delay.

In this section, we present in some detail the modelling needs in the following three directions:

- take into account different ways to collect data on the state of traffic;

- look for more accurate descriptions of capacity drop, including memory effects and self-organization;

- in particular, introduce proper dynamics of evolution of the subjective density (inertia, etc.).

Numerical simulations illustrating the relevant features of advanced models put forward in this section are postponed to Section 7

Let us introduce the maps $p:\left[0, \rho_{\text {max }}\right] \rightarrow\left(0, f_{\text {max }}\right], w: \mathbb{R}_{-} \rightarrow \mathbb{R}_{+}$and $\kappa: \mathbb{R}_{+} \rightarrow \mathbb{R}_{+}$that will be used throughout the paper to express, respectively, the dependence of the constraint level on the subjective density, the space non-locality and the time non-locality (memory). For simplicity, let us assume that

$(\mathbf{P W K})$

- $p \in \operatorname{Lip}\left(\left[0, \rho_{\max }\right] ;\left(0, f_{\max }\right]\right)$ is a non-increasing map;

- $w \in \mathbf{L}^{\infty}\left(\mathbb{R}_{-} ; \mathbb{R}_{+}\right)$is a non-decreasing map with $\|w\|_{\mathbf{L}^{\mathbf{1}\left(\mathbb{R}_{-}\right)}}=1$ and $\operatorname{supp}(w)=\left[-\mathrm{i}_{w}, 0\right], \mathrm{i}_{w}>0$;

- $\kappa \in \operatorname{Lip}\left(\mathbb{R}_{+} ; \mathbb{R}_{+}\right)$is a non increasing map with $\|\kappa\|_{\mathbf{L}^{\mathbf{1}\left(\mathbb{R}_{+}\right)}}=1$ and $\operatorname{supp}(\kappa)=[0, \tau], \tau>0$.

Whenever $w^{\prime}$ appears in the expression of a constraint operator, wLip below is assumed as well.

The above assumptions can be altered to varying degrees in each specific modelling context, with minor consequences on the subsequent results.

1.4.1. Different modes of data acquisition. Let $p, w$ and $\kappa$ be as in $(\overline{\mathbf{P W K}})$ with $w \in \mathbf{C}_{\mathbf{c}}^{\mathbf{l}}\left(\mathbb{R} ; \mathbb{R}_{+}\right)$. Consider the following equivalent (in a sense that will be specified in Definition 2.2 non-local (both in time and space) constraint operators

$$
\begin{aligned}
& \mathscr{Q}[\rho](t)=p\left(\int_{\mathbb{R}_{-}} \int_{0}^{t} w(x) \kappa(t-s) \rho(s, x) \mathrm{d} s \mathrm{~d} x\right), \\
& \widehat{\mathscr{Q}}[\rho](t)=p\left(\int_{\mathbb{R}_{-}}\left[w(x) \bar{\kappa}(t) \rho_{0}(x)+w^{\prime}(x) \int_{0}^{t} \bar{\kappa}(t-s) f(\rho)(s, x) \mathrm{d} s\right] \mathrm{d} x-w\left(0^{-}\right) \int_{0}^{t} \bar{\kappa}(t-s) f(\rho)\left(s, 0^{-}\right) \mathrm{d} s\right),
\end{aligned}
$$

where $\bar{\kappa} \in \mathbf{C}^{2}\left(\mathbb{R}_{+} ; \mathbb{R}_{+}\right)$is the primitive of $\kappa$ such that $\bar{\kappa}(0)=0$.

The constraint operators $\mathscr{Q}$ and $\widehat{\mathscr{Q}}$ reproduce the situation in which the maximal flow at time $t$ in $x=0$ depends on the values of the density in $\operatorname{supp}(\kappa(t-\cdot)) \times \operatorname{supp}(w)$. Moreover, due to the monotonicity assumptions on $w$, respectively on $\kappa$, the efficiency is more affected by the "closest" values of the density, respectively by the "more recent" 
values of the density. Introduce also the constraint operators

$$
\begin{aligned}
& \widetilde{\mathscr{Q}}[\rho](t)=p\left(\sum_{0<t_{i} \leq t}\left[t_{i}-t_{i-1}\right] \kappa\left(t-t_{i-1}\right) \int_{\mathbb{R}_{-}} w(x) \rho\left(t_{i}, x\right) \mathrm{d} x\right), \\
& \overline{\mathscr{Q}}[\rho](t)=p\left(\sum_{y_{i}<0}\left[y_{i+1}-y_{i}\right] w\left(y_{i}\right)\left[\int_{0}^{t} \kappa(t-s) \rho\left(s, y_{i+1}\right) \mathrm{d} s\right]\right),
\end{aligned}
$$

which can be interpreted as discretized versions of $\mathscr{Q}$, with $0 \leq t_{i}<t_{i+1}$ and $y_{0} \leq y_{i}<y_{i+1} \leq y_{M+1}=0$.

Constraint operators of the form introduced above naturally arise in modelling vehicular traffics through a toll booth if the number of open gates is decided according to on line data. In fact, we have the following:

- In the case the data are collected by a video camera, then the corresponding constraint operator takes the form $\mathscr{Q}$ or $\widehat{\mathscr{Q}}$, being $\operatorname{supp}(w)$ the area registered by the video camera and $\operatorname{supp}(\kappa)$ the period of time the data are taken into account.

- In the case the data come from a photo camera that shoots photos at times $t_{i}$ of the area given by $\operatorname{supp}(w)$, then the corresponding constraint operator is $\widetilde{\mathscr{Q}}$, being again $\operatorname{supp}(\kappa)$ the period of time the data are taken into account.

- In the case the data come from local sensors located in $y_{i}$, then the corresponding constraint operator takes the form $\overline{\mathscr{Q}}$, being again $\operatorname{supp}(\kappa)$ the period of time the data are taken into account.

Also observe that the introduction of a time delay $\sigma>0$ in adapting the number of open gates to the available data can be modelled, for instance, by taking

$$
\mathscr{Q}[\rho](t)=p\left(\int_{\mathbb{R}_{-}} \int_{0}^{\max \{0, t-\sigma\}} w(x) \kappa(t-\sigma-s) \rho(s, x) \mathrm{d} s \mathrm{~d} x\right) .
$$

The numerical simulations based on the models introduced hereabove are deferred to Example 7.1 where we compare the resulting numerical simulations corresponding to the different models. In particular we show that the simulations corresponding to $\mathscr{Q}$ and $\widehat{\mathscr{Q}}$ are essentially the same. Also the simulations with $\widetilde{\mathscr{Q}}$ and $\overline{\mathscr{Q}}$ are essentially the same as those with $\mathscr{Q}$ and $\widehat{\mathscr{Q}}$ if the number of times $t_{i}$ and of sensors $y_{i}$ is very high.

1.4.2. More accurate modeling of capacity drop. Memory effects and self-organization. In 4 the authors consider the constraint operator

$$
\mathscr{Q}[\rho](t)=p(\xi(t))
$$

$$
\xi(t)=\int_{\mathbb{R}_{-}} w(x) \rho(t, x) \mathrm{d} x
$$

where $p$ and $w$ are given by $\overline{\text { PWK }}$, to reproduce the capacity drop of a bottleneck when a very high density of pedestrians accumulate upstream (see also the examples 2.1 and 4.1. However this model is not realistic if the density contains big oscillations. In fact, as soon as a high density approaches the bottleneck, its efficiency can become very small. As a consequence, even a small density of pedestrians may form a queue provided a sufficiently high density is approaching from behind. In order to temper this drawback, we propose a constraint operator of the form

$$
\mathscr{Q}[\rho](t)=p(\xi(t)), \quad \xi(t)=\min \left\{\int_{\mathbb{R}_{-}} w(x) \rho(t, x) \mathrm{d} x, \alpha f_{-}^{-1}\left(\int_{0}^{t} \kappa(t-s) f(\rho)\left(s, 0^{-}\right) \mathrm{d} s\right)\right\},
$$

where $0<\alpha \leq \frac{R}{\rho_{c}}$ is a constant, $p, w$ and $\kappa$ are as in (PWK and $f_{-}$is the restriction of $f$ to [0, $\rho_{c}$ ]. In [1.6], the maximal flow at time $t$ in $x=0$ depends at the same time on the weighted number of pedestrians located in a neighbourhood before the bottleneck and on the weighted number of pedestrians that have passed through the bottleneck during the period $[0, t]$, i.e., a kind of memory is taken into account. In fact, when a density of pedestrian approaches the bottleneck, the constraint operator defined in (1.6) first takes into account the flow of pedestrians through the bottleneck. Next, because the number of pedestrians before the bottleneck decreases in time, this latter is taken into account by the constraint operator in a second step. The two numerical simulations given in Example 7.2 (see Figure 6) with the same initial datum and map $w$, but one with the constraint operator (1.5) and one with the constraint operator (1.6), show that at least in this test case the constraint operator [1.6] does not present the drawback pointed out hereabove.

At the same time and on the contrary to [1.5], the constraint operator [1.6) is able to simulate the self-organization of pedestrians and the partial restoration of the capacity of the bottleneck, see [51 Figure 3] for empirical data detected in a London underground station that put in evidence both these aspects. The resulting numerical simulations are presented in Example 7.2 (see Figure 7). Let us underline that the same approach can be used to model analogous dynamics occurring in vehicular traffic through bottlenecks, see [51, Figure 4]. Clearly, the choice of the functions $w$ and $\kappa$ should take into account the empirical data, and mechanisms underlying self-organization should be related to observation of real queues. Accurate reproduction of self-organization features is a particularly challenging issue, which goes beyond the aims of the present paper. 
1.4.3. Proper dynamics of the subjective density. In the model [1.1, [1.3, , 1.5 introduced in [4], the function $\xi$ serves as the indicator of the subjective density at the bottleneck and it is readily checked that it is the solution of a Cauchy problem for the Ordinary Differential Equation (ODE) $\dot{\xi}(t)=\int_{\mathbb{R}_{-}} w^{\prime}(x)\left[f(\rho)(t, x)-f(\rho)\left(t, 0^{-}\right)\right] \mathrm{d} x$, see [4, page 2688] and 2.3. Thus in ADR, $\xi$ possesses a dynamics that is governed uniquely by the instantaneous values of the density $\rho$ coming from LWR. However, in the context of crowd dynamics, memory effects of inertia kind should be taken into account (in a sense, the panic underlying the irrational behavior and the capacity drop cannot decay abruptly). Introducing a dynamics of its own, in order to describe in particular the inertial effects, is another way to enrich ADR. As an example, we can model the fact that the panic can not disappear too fast by considering the constraint operator

$$
\widehat{\mathscr{Q}}[\rho](t)=p(\xi(t)),
$$

where $\xi$ is defined as the solution in $\mathscr{D}^{\prime}([0, T))$ of the following Cauchy problem for an ODE

$$
\dot{\xi}(t)=\max \left\{\int_{\mathbb{R}_{-}} w^{\prime}(x)\left[f(\rho)(t, x)-f(\rho)\left(t, 0^{-}\right)\right] \mathrm{d} x,-\delta \xi\right\}, \quad \xi(0)=\xi_{0}\left[\rho_{0}\right],
$$

or

$$
\dot{\xi}(t)=\max \left\{\int_{\mathbb{R}_{-}} w^{\prime}(x)\left[f(\rho)(t, x)-f(\rho)\left(t, 0^{-}\right)\right] \mathrm{d} x,-\delta\right\}, \quad \xi(0)=\xi_{0}\left[\rho_{0}\right],
$$

where $\xi_{0}: \mathbf{L}^{\mathbf{1}}(\mathbb{R}) \rightarrow \mathbb{R}, \delta>0$ is a constant and $w: \mathbb{R}_{-} \rightarrow \mathbb{R}_{+}$is such that

$$
w \in \operatorname{Lip}\left((-\infty, 0) ; \mathbb{R}_{+}\right) \text {but it can be/is discontinuous at } x=0 .
$$

This has to be compared to the original model [1.5], cf. Example 2.1 below. We defer to Remark 4.3 for an existence and uniqueness result for [1.1, 1.3, [1.7, , 1.8. By the numerical simulation in Example 7.3 we see that the constraint operator (1.7) with 1.8 or $(1.9)$ is able to avoid the level of subjective density to decrease too quickly.

1.5. Structure of the paper. The paper is organized as follows. In Section 2 we introduce the general assumptions on the constraint operators that allow to prove well-posedness of 1.1 and we recall the definition of entropy solutions for (1.1). In Section 3 we prove well-posedness results for [1.1) under the assumptions introduced in Section 2 In Section 4 we give examples of constraint operators that satisfy the general assumptions introduced in the previous sections and that can be used as building blocks to construct more complex ones. In Section 5 we introduce the numerical scheme, based on finite volume methods and we prove its convergence. Section 6 is devoted to the construction and the study of approximate constraint operators related to the constraint operators introduced in Section 4. Finally in Section 7, we first validate the numerical scheme with an explicit solution and then we perform numerical simulations using constraint operators given in Sections $1.4 .1-1.4 .3$

\section{ASSUMPTIONS AND DEFINITIONS}

Our first goal is to point out properties of the constraint operators $\mathscr{Q}$ for which a sound mathematical theory can be established.

2.1. Assumptions. We take the classical assumption $[\mathbf{F}$ on the flux function $f$ that appears in the equation $1.1 \mathrm{a}$. Existence results will be stronger under the additional genuine nonlinearity assumption:

(GNL)

$$
\text { for any } \rho_{1}, \rho_{2} \in\left[0, \rho_{\max }\right] \text { such that } \rho_{1}<\rho_{2} \text {, the restriction of } f \text { to }\left[\rho_{1}, \rho_{2}\right] \text { is not affine. }
$$

For the sake of simplicity and because this is enough for our modelling purposes, we will impose that the initial datum $\rho_{0}$ that appears in $\left[1.1 \mathrm{c}\right.$ ) belongs to $\mathbf{L}^{\mathbf{1}}\left(\mathbb{R} ;\left[0, \rho_{\max }\right]\right)$. Let us underline that, thanks to the property of finite speed of propagation for equation [1.1a], extension of the existence and uniqueness results to $\mathbf{L}^{\infty}\left(\mathbb{R} ;\left[0, \rho_{\max }\right]\right)$ is rather straightforward (see, in particular, the proof of [4. Theorem 2.1]).

Now, let us specify properties of the operator $\mathscr{Q}$. Firstly, we need an operator acting as follows

$$
\mathscr{Q}: \mathbf{C}^{\mathbf{0}}\left(0, T ; \mathbf{L}^{\mathbf{1}}\right) \longrightarrow \mathbf{L}^{\mathbf{1}}(0, T),
$$

where

$$
\mathbf{C}^{\mathbf{0}}\left(0, T ; \mathbf{L}^{\mathbf{1}}\right)=\mathbf{C}^{\mathbf{0}}\left([0, T] ; \mathbf{L}^{\mathbf{1}}\left(\mathbb{R} ;\left[0, \rho_{\max }\right]\right)\right) \quad \text { and } \quad \mathbf{L}^{\mathbf{l}}(0, T)=\mathbf{L}^{\mathbf{1}}\left((0, T) ;\left[0, f_{\max }\right]\right)
$$

are endowed, respectively, with the distances induced by the norms

$$
\|\rho\|_{\mathbf{C}^{\mathbf{0}}\left(0, T ; \mathbf{L}^{\mathbf{1}}\right)}=\max _{t \in[0, T]} \int_{\mathbb{R}}|\rho(t, x)| \mathrm{d} x \quad \quad \text { and } \quad\|q\|_{\mathbf{L}^{\mathbf{1}}(0, T)}=\int_{0}^{T}|q(t)| \mathrm{d} t .
$$

On concrete examples, the technical assumptions imposed below may be easier to verify as consequences of some reinforced assumptions involving $\mathbf{L}^{\mathbf{1}}\left(0, T ; \mathbf{L}^{\mathbf{1}}\right)$ or $\mathbf{L}^{\infty}(0, T)$ in the place of $\mathbf{C}^{\mathbf{0}}\left(0, T ; \mathbf{L}^{\mathbf{1}}\right)$ and $\mathbf{L}^{\mathbf{1}}(0, T)$, where

$$
\mathbf{L}^{\mathbf{1}}\left(0, T ; \mathbf{L}^{\mathbf{1}}\right)=\mathbf{L}^{\mathbf{1}}\left((0, T) \times \mathbb{R} ;\left[0, \rho_{\max }\right]\right) \quad \text { and } \quad \mathbf{L}^{\infty}(0, T)=\mathbf{L}^{\infty}\left((0, T) ;\left[0, f_{\max }\right]\right)
$$


are endowed, respectively, with the distances induced by the norms

$$
\|\rho\|_{\mathbf{L}^{\mathbf{1}}\left(0, T ; \mathbf{L}^{\mathbf{1}}\right)}=\int_{0}^{T} \int_{\mathbb{R}}|\rho(t, x)| \mathrm{d} x \mathrm{~d} t \quad \quad \text { and } \quad\|q\|_{\mathbf{L}^{\infty}(0, T)}=\operatorname{ess}_{\sup _{t \in(0, T)}|q(t)| .}
$$

For this reason, whenever necessary, we assume that the constraint operator $\mathscr{Q}$ can be extended to $\mathbf{L}^{\mathbf{1}}\left(0, T ; \mathbf{L}^{\mathbf{l}}\right)$. Observe that the embeddings $\mathbf{L}^{\mathbf{1}}\left(0, T ; \mathbf{L}^{\mathbf{l}}\right) \supset \mathbf{C}^{\mathbf{0}}\left(0, T ; \mathbf{L}^{\mathbf{1}}\right)$ and $\mathbf{L}^{\infty}(0, T) \subset \mathbf{L}^{\mathbf{1}}(0, T)$ are continuous.

The first natural assumption is that $\mathscr{Q}$ is "history dependent" (cf. 49] for a related work on history-dependent operators in the setting of viscoplastic contact problems) in the sense that

$\left(\mathbf{Q}^{\text {hd }}\right)$

$$
\rho_{1}, \rho_{2} \text { are in the domain of } \mathscr{Q} \text { and coincide on }[0, t] \times \mathbb{R} \Longrightarrow \mathscr{Q}\left[\rho_{1}\right]=\mathscr{Q}\left[\rho_{2}\right] \text { in }[0, t] .
$$

This simply means that the constraint level does not depend on the future traffic but only on the past and actual traffic, therefore it is a fully natural assumption in applications (even if the existence result may not require assumption $\left.\mathbf{Q}^{\mathrm{hd}}\right)$. In particular, assuming $\mathbf{Q}^{\mathrm{hd}}$ one can introduce for any $t \in[0, T]$ the restriction of $\mathscr{Q}$ to $\mathbf{C}^{\mathbf{0}}\left(0, t ; \mathbf{L}^{\mathbf{l}}\right)$ as the operator $\mathscr{Q}_{t}: \mathbf{C}^{\mathbf{0}}\left(0, t ; \mathbf{L}^{\mathbf{1}}\right) \rightarrow \mathbf{L}^{\mathbf{1}}(0, t)$ defined as follows. First, for any given function $\rho \in \mathbf{C}^{\mathbf{0}}\left(0, t ; \mathbf{L}^{\mathbf{1}}\right)$ one introduces an arbitrary extension $\mathscr{E}[\rho] \in \mathbf{C}^{\mathbf{0}}\left(0, T ; \mathbf{L}^{\mathbf{1}}\right)$, say, by letting $\mathscr{E}[\rho](s)=\rho(s, \cdot) \mathbf{1}_{[0, t]}(s)+\rho(t, \cdot) \mathbf{1}_{[t, T]}(s)$. Then, one defines $\mathscr{Q}_{t}[\rho]$ as the restriction to $[0, t]$ of $\mathscr{Q}[\mathscr{E}[\rho]]$.

In the sequel, whenever necessary we will tacitly use the possibility of such restriction of $\mathscr{Q}$ and denote the resulting operator by $\mathscr{Q}_{t}$. Yet, property $\mathbf{Q}^{\text {hd }}$ will not explicitly appear in our assumptions, because it is either too weak or too strong for our purposes. Indeed, in view of the uniqueness study, assumption $\mathbf{Q}^{\text {hd }}$ has to be strengthened to a kind of Lipschitz continuity assumption on $\mathscr{Q}_{t}$, with Lipschitz constant that is small for small times. The precise assumption is technical and reads as follows:

for any $t \in[0, T]$ the operator $\mathscr{Q}_{t}: \mathbf{C}^{\mathbf{0}}\left(0, t ; \mathbf{L}^{\mathbf{1}}\right) \rightarrow \mathbf{L}^{\mathbf{1}}(0, t)$ is Lipschitz continuous, moreover, there exists $\omega: \mathbb{R}_{+} \rightarrow \mathbb{R}_{+}$continuous, non-decreasing, $\omega(0)=0$ and for any $\rho_{1}, \rho_{2} \in \mathbf{C}^{\mathbf{0}}\left(0, T ; \mathbf{L}^{\mathbf{1}}\right)$

$\left(\mathbf{Q}^{\mathrm{Lip}}\right)$

$$
\left\|\mathscr{Q}_{t}\left[\rho_{1}\right]-\mathscr{Q}_{t}\left[\rho_{2}\right]\right\|_{\mathbf{L}^{\mathbf{1}}(0, t)} \leq \omega(t-\tau)\left\|\rho_{1}-\rho_{2}\right\|_{\mathbf{C}^{\mathbf{0}}\left(0, t ; \mathbf{L}^{\mathbf{1}}\right)}
$$

for a.e. $t \in[0, T]$,

where $\tau=\max \left\{s \in[0, t]: \rho_{1}=\rho_{2}\right.$ in $\left.\mathbf{C}^{\mathbf{0}}\left(0, s ; \mathbf{L}^{\mathbf{1}}\right)\right\}$.

Under assumption $\mathbf{Q}^{\text {Lip }}$, the Banach-Picard fixed point argument can be used recursively on small time intervals to yield both existence and uniqueness of solutions. There are simpler sufficient conditions under which $\mathbf{Q}^{\mathrm{Lip}}$ holds, see [3.4, 3.5 and Corollary 3.1 in Section 3.2. They take the form of uniform in $t$ Lipschitz continuity assumptions between the appropriate spaces; these assumptions are easily checked for the examples in Section 4

Let us underline that for the existence result, assumption $\mathbf{Q}^{\mathrm{Lip}}$ is sufficient but in general it is too restrictive. Moreover, $\mathbf{Q}^{\mathrm{Lip}}$ should be used in combination with the Lipschitz estimate 3.3 stated in Lemma 3.2 below. The result of this lemma holds true for the scalar constrained Cauchy problem (1.1). Note that the theory of point constraints has recently been applied to systems of conservation laws as well, see for instance [5]; in the context of systems, results analogous to those of Lemma 3.2 can hardly be proved. In further perspective, we are interested in studying such more complex models, at least from the perspective of existence and approximation of their solutions. For this reason we observe that under assumption GNL it is enough to require the mere continuity property of an extension of $\mathscr{Q}$, namely

$\left(\mathbf{Q}^{\text {cont }}\right) \quad \mathscr{Q}$ can be extended to a continuous map from $\mathbf{L}^{\mathbf{1}}\left(0, T ; \mathbf{L}^{\mathbf{1}}\right)$ to $\mathbf{L}^{\mathbf{1}}(0, T)$.

In the situation where the genuine nonlinearity assumption $\mathbf{G N L}$ should be dropped, we can replace $\mathbf{Q}^{\text {cont }}$ by

$$
\mathscr{Q} \text { is continuous and compact from } \mathbf{C}^{\mathbf{0}}\left(0, T ; \mathbf{L}^{\mathbf{1}}\right) \text { to } \mathbf{L}^{\mathbf{1}}(0, T) \text {. }
$$

Because the topology of $\mathbf{L}^{\mathbf{1}}\left(0, T ; \mathbf{L}^{\mathbf{1}}\right)$ is weaker that the one of $\mathbf{C}^{\mathbf{0}}\left(0, T ; \mathbf{L}^{\mathbf{1}}\right)$, the two assumptions $\mathbf{Q}^{\text {cont }}$ and $\mathbf{Q}^{\text {comp }}$

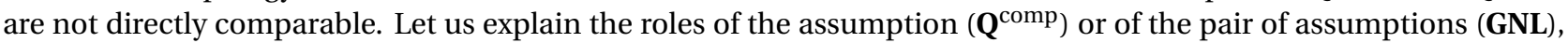
$\mathbf{Q}^{\text {cont }}$. In the latter case, compactness of families of functions $\left(\rho_{\Delta}\right)$ solving [1.1a) away from $x=0$ can be justified by using the compactification properties of genuinely nonlinear scalar conservation law, see 44]. The appropriate topology is the $\mathbf{L}^{\mathbf{1}}$ topology, which requires to use the space $\mathbf{L}^{\mathbf{1}}\left(0, T ; \mathbf{L}^{\mathbf{l}}\right)$ in the place of the more natural $\operatorname{space}^{\mathbf{0}}\left(0, T ; \mathbf{L}^{\mathbf{1}}\right)$. Under the assumption $\mathbf{Q}^{\text {comp }}$, compactness of the corresponding constraints $\left(q_{\Delta}\right)$ (and consequently that of $\left(\rho_{\Delta}\right)$ ) is a straightforward property. In both cases, the Schauder fixed point argument can be used in order to resolve the coupling between $\rho$ and $q$ in $\left[1.1,1.3\right.$. Assumption $\mathbf{Q}^{\text {comp }}$ will be also taken for the study of numerical approximations of the problem, for the sake of simplicity of the proof. We refer to Remark 5.2 for details.

2.2. Entropy solutions for models with point constraints. We are ready to define a notion of solution for the equation (1.1a) subject to a nonlocal point constraint. We define the solution on $[0, T]$ for $T>0$; extension of the definition to $t \in \mathbb{R}_{+}$is straightforward. Throughout the paper, assumptions on $f$ and $\rho_{0}$ are those of the introduction, except for GNL which will be mentioned explicitly each time it is used.

For our purposes, it is convenient to consider the problem as a $2 \times 2$ system of equations for the two unknowns $\rho$ and $q$. We split the definition into two points, the first one being applicable to the case where $q$ is a given function on $[0, T]$. 
Definition 2.1. A couple $(\rho, q)$ such that $\rho \in \mathbf{C}^{\mathbf{0}}\left([0, T] ; \mathbf{L}_{\mathbf{l o c}}^{\mathbf{1}}\left(\mathbb{R} ;\left[0, \rho_{\max }\right]\right)\right)$ and $q \in \mathbf{L}^{\infty}(0, T)$ is an entropy solution to $[1.1]$, 1.3. if the following conditions hold.

(i) The function $\rho$ is an entropy solution of the constrained Cauchy problem [1.1] with the constraint q, i.e., for every test function $\phi \in \mathbf{C}_{\mathbf{c}}^{\infty}\left([0, T) \times \mathbb{R} ; \mathbb{R}_{+}\right)$and for every $k \in\left[0, \rho_{\max }\right]$

$$
\begin{array}{r}
\int_{\mathbb{R}_{+}} \int_{\mathbb{R}}\left[|\rho-k| \partial_{t} \phi+\operatorname{sign}(\rho-k)(f(\rho)-f(k)) \partial_{x} \phi\right] \mathrm{d} x \mathrm{~d} t \\
+2 \int_{\mathbb{R}_{+}}\left[1-\frac{q(t)}{f\left(\rho_{c}\right)}\right] f(k) \phi(t, 0) \mathrm{d} t \\
+\int_{\mathbb{R}}\left|\rho_{0}(x)-k\right| \phi(0, x) \mathrm{d} x \geq 0,
\end{array}
$$

and the left and right traces $t \mapsto \gamma^{ \pm} f(\rho)(t)$ of $f(\rho)$ at $\{x=0\}$ fulfill

$$
\gamma^{ \pm} f(\rho)(t) \leq q(t) \text { for a.e. } t \in[0, T] .
$$

(ii) The function $q$ is linked to $\rho$ by the relation 1.3 .

Recall that the item (i) is precisely [6, Definition 2.1], which is a minor generalization of the original one [21] Definition 3.2] introduced by Colombo and Goatin. Let us stress that the lines [2.1a], 2.1c] constitute the classical Kruzhkov definition of an entropy solution, see [39], suitable in the case of a conservation law set up in $\mathbb{R}_{+} \times \mathbb{R}$ without any constraint condition, namely for (1.1a), 1.1c). Lines [2.1b) and (2.1d) account for the constraint (1.1b).

\section{Remark 2.1.}

(i) In order to give a precise meaning to 2.1d, let us stress that strong (in the $\mathbf{L}_{\text {loc }}^{\mathbf{1}}$ sense) left and right traces at $x=0$ of the flux of a function verifying [2.1a) - 2.1c do exist. We refer, for instance, to [6. Theorem 2.2] which gives a convenient reformulation of the results of Vasseur [50] and Panov [45].

We also stress that in practical applications where $f$ is non-constant on any interval (assumption $\mathbf{G N L}$ is a sufficient condition) $f(\rho)\left(t, 0^{ \pm}\right)$coincides with $f\left(\rho\left(t, 0^{ \pm}\right)\right)$. Indeed, in this case the strong traces $t \mapsto \gamma^{ \pm} \rho(t)$ exist as well, see [45]. In the sequel, we will write $f(\rho)\left(t, 0^{ \pm}\right)$for $\gamma^{ \pm} f(\rho)(t)$ and $\rho\left(\cdot, 0^{ \pm}\right)$for $\gamma^{ \pm} \rho(t)$.

(ii) Let us recall that the assumption of time-continuity (with values in $\mathbf{L}_{\text {loc }}^{\mathbf{l}}(\mathbb{R})$ ) of entropy solutions in the sense of Definition 2.1 (i) is a regularity property of general $\mathbf{L}_{\text {loc }}^{\mathbf{1}}((0, T) \times \mathbb{R})$ solutions, analogous to the above property of existence of traces. This regularity is justified, e.g., in [15].

(iii) We refer to [6, Proposition 2.6] for a series of equivalent formulations of conditions [2.1 that we will not exploit directly in this paper, although the results we exploit in the sequel (continuous dependence of $\rho$ on $q$, convergence of numerical schemes for a given $q$ ) do rely upon the reformulations from [6].

It turns out that the constrained problems of the form (1.1), [1.3) corresponding to two different constraint operators $\mathscr{Q}$ and $\widehat{\mathscr{Q}}$ may lead to the same solutions for all data. In this context, the mathematical properties of one of the constraint operators can be better than those of the other one, which is sometimes instrumental for the analysis. For this reason, we introduce and exploit the following definition.

Definition 2.2. Two constraint maps $\mathscr{Q}$ and $\widehat{\mathscr{Q}}$ are equivalent if $\mathscr{Q}[\rho]=\widehat{\mathscr{Q}}[\rho]$ for any entropy solution $\rho$ of $[1.1$, , 1.3$]$ corresponding to $\mathscr{Q}$ or to $\widehat{\mathscr{Q}}$.

Example 2.1. Consider the constraint operator $\mathscr{Q}$ introduced in [4] and defined in [1.5] with $p$ and $w$ that satisfy $(\mathbf{P W K}$. Assume in addition that $w$ satisfies $\mathbf{\text { wLip }}$ and introduce also the constraint operator

$$
\widehat{\mathscr{Q}}[\rho](t)=p(\xi(t)),
$$

where $\xi$ is the solution in $\mathscr{D}^{\prime}([0, T))$ of the following Cauchy problem for an ODE

$$
\dot{\xi}(t)=\int_{\mathbb{R}_{-}} w^{\prime}(x)\left[f(\rho)(t, x)-f(\rho)\left(t, 0^{-}\right)\right] \mathrm{d} x, \quad \xi(0)=\int_{\mathbb{R}_{-}} w(x) \rho_{0}(x) \mathrm{d} x .
$$

Then the constraint operators $\mathscr{Q}$ and $\widehat{\mathscr{Q}}$ are equivalent in the sense of Definition 2.2 . Indeed, for any entropy solution $(\rho, q)$ to (1.1), (1.3), (1.5) or to [1.1], (1.3), 2.2), 2.3, $\rho$ satisfies the Cauchy problem for a conservation law (1.1a), 1.1c) in $\mathscr{D}^{\prime}([0, T) \times \mathbb{R})$ (this is a consequence of 2.1a) $-2.1 \mathrm{c}$ with $k=0$ and $\left.k=\rho_{\max }\right)$. It is evident that, given $(\rho, q)$ entropy solution to the constrained Cauchy problem [1.1], [1.3, , 1.5, there exists a unique solution $\xi$ to problem [2.3. Then, by applying the integration by parts formula to the right-hand side of the ODE in [2.3] we find

$$
\dot{\xi}(t)=-\int_{\mathbb{R}_{-}} w(x) f(\rho)_{x}(t, x) \mathrm{d} x \quad \text { in } \mathscr{D}^{\prime}((0, T)),
$$

hence by (1.1a), 1.1c) and by taking into account the initial condition in $[2.3$, we see that $\xi$ coincides in $[0, T]$ with $\xi$ given in 1.5 and

$$
\widehat{\mathscr{Q}}[\rho]=p(\xi(t))=p\left(\int_{\mathbb{R}_{-}} w(x) \rho(t, x) \mathrm{d} x\right)=\mathscr{Q}[\rho] .
$$


Reciprocally, taking the time derivative of 1.5 in the sense of distributions, with the same arguments we infer 2.2, 2.3. We defer to Example 4.1 for a deeper study of the constraint operator $\mathscr{Q}$ and to Example 6.1 for the study of a corresponding discrete constraint operator. Finally, we defer to Remark 4.2 for an existence and uniqueness result for [1.1, 1.3, 2.2, 2.3.

Example 2.2. Take $p$ and $\kappa$ as in $(\mathbf{P W K})$ and $\phi \in \operatorname{Lip}\left([0, T] ; \mathbf{C}_{\mathbf{c}}^{\mathbf{l}}(\mathbb{R} ; \mathbb{R})\right)$ such that $\phi\left(\cdot, 0^{-}\right)=\kappa(\cdot)$. Take in addition $r \in$ $\operatorname{Lip}\left(\left[0, f_{\text {max }}\right] ;\left[0, \rho_{\max }\right]\right)$. The constraint operators

$$
\mathscr{Q}[\rho](t)=p \circ r\left(\int_{0}^{t} f(\rho)\left(s, 0^{-}\right) \kappa(t-s) \mathrm{d} s\right) \quad \text { and } \quad \widetilde{\mathscr{Q}}[\rho](t)=p \circ r(\xi(t)),
$$

where

$$
\begin{aligned}
\xi(t)= & \int_{0}^{t} \int_{\mathbb{R}_{-}}\left[-\rho(s, x) \phi_{t}(t-s, x)+f(\rho)(s, x) \phi_{x}(t-s, x)\right] \mathrm{d} x \mathrm{~d} s \\
& +\int_{\mathbb{R}_{-}}\left[\rho_{0}(x) \phi(t, x)-\rho(t, x) \phi(0, x)\right] \mathrm{d} x,
\end{aligned}
$$

are equivalent. As in the example above, the equivalence is established by using integration by parts formula and the fact that $\rho$ satisfies the Cauchy problem for a conservation law $1.1 \mathrm{a}),\left[1.1 \mathrm{c}\right.$ in $\mathscr{D}^{\prime}([0, T) \times \mathbb{R})$. We refer to Example 4.3 for the study of an analogous example.

Observe that the definition of $\xi$ in terms of the averaged trace of the flux $f\left(\rho\left(0^{-}, \cdot\right)\right)$ permits to recover stability properties of the constraint operator due to the Green-Gauss formula. In contrast, time averaging of the trace $\rho\left(0^{-}, \cdot\right)$ does not lead to a consistent mathematical theory. For this reason, in the present example we need to introduce a function $r$ that plays the role of a partial inverse of $f$. To be specific, let us denote by $f_{-}$(respectively, by $f_{+}$) the restriction of $f$ to $\left[0, \rho_{c}\right]$ (respectively, to $\left[\rho_{c}, \rho_{\text {max }}\right]$ ). Observe that the model of this example can be seen as qualitatively close to 1.5 for sufficiently large crowd densities (respectively, for moderate crowd densities), if we choose $r$ that behaves like $f_{+}^{-1}$ (respectively, as $f_{-}^{-1}$ ) on some interval $[0, F]$ with $F<f_{\text {max }}$. Compared to 1.5 where the subjective density results from the perception of the instantaneous spatial distribution of the crowd, in this example the subjective density results from the memory of the crowd behavior at the precise location of the bottleneck.

\section{EXISTENCE AND UNIQUENESS OF ENTROPY SOLUTIONS TO CONSTRAINED PROBLEMS}

In this section, using fixed point arguments we show existence results (under the assumptions $\left(\mathbf{G N L}\right.$ and $\left(\mathbf{Q}^{\text {cont }}\right.$ or under the assumption $\mathbf{Q}^{\text {comp }}$ ) and uniqueness results (under the assumption $\mathbf{Q}^{\mathrm{Lip}}$ ).

3.1. Evolution operators. To start with, let us define the following operators. Here and throughout the section, the initial datum $\rho_{0}$ is fixed. The maps

$$
\mathscr{R}_{t}: \mathbf{L}^{\mathbf{1}}(0, t) \rightarrow \mathbf{C}^{\mathbf{0}}\left(0, t ; \mathbf{L}^{\mathbf{1}}\right)
$$

associate to a given constraint function $q$ the entropy solution $\rho$ to (1.1) with time horizon $t$ in the sense of Definition 2.1 (i). Composing this map with $\mathscr{Q}_{t}$, we define the map

$$
\mathscr{T}_{t}=\mathscr{R}_{t} \circ \mathscr{Q}_{t}: \mathbf{C}^{\mathbf{0}}\left(0, t ; \mathbf{L}^{\mathbf{1}}\right) \rightarrow \mathbf{C}^{\mathbf{0}}\left(0, t ; \mathbf{L}^{\mathbf{1}}\right) .
$$

Let us stress that $t$ is not necessarily fixed to be equal to $T$, namely, under the history dependence assumption $\mathbf{Q}^{\text {hd }}$, we can consider first sufficiently small time horizon and then proceed by bootstrapping the construction of entropy solutions in order to achieve the prescribed final time $T$. To be precise, we have

Lemma 3.1. Assume that the constraint operator $\mathscr{Q}$ verifies property $\mathbf{Q}^{\text {hd }}$. Let $0=t_{0}<t_{i}<t_{i+1}<t_{N}=T$ and let $\left(\rho^{(1)}, q^{(1)}\right), \ldots,\left(\rho^{(N)}, q^{(N)}\right)$ be defined recursively as follows. First,

$\left(\rho^{(1)}, q^{(1)}\right)$ is an entropy solution in the sense of Definition 2.1 to the constrained Cauchy problem 1.1 , , 1.3 with initial datum $\rho_{0}$, constraint operator $\mathscr{Q}$ and time horizon $t_{1}$.

Then for $i=2, \ldots, N$, we set $\rho_{0}^{(i)}=\rho^{(i-1)}\left(t_{i-1}-t_{i-2}\right)$ and for $s \in\left[0, t_{i}-t_{i-1}\right], q^{i}(s):=\mathscr{Q}^{(i)}[\rho](s)=\mathscr{Q}\left[\mathscr{E}^{(i)}[\rho]\right]\left(s+t_{i-1}\right)$, where 1

$$
\mathscr{E}^{(i)}[\rho](t):=\rho_{0} \mathbf{1}_{\{0\}}(t)+\sum_{j=1}^{i-1} \rho^{(j)}\left(t-t_{j-1}\right) \mathbf{1}_{\left(t_{j-1}, t_{j}\right]}(t)+\rho\left(t-t_{i-1}\right) \mathbf{1}_{\left(t_{i-1}, t_{i}\right]}(t) .
$$

With these notations in hand, we assume that

$\left(\rho^{(i)}, q^{(i)}\right)$ is an entropy solution in the sense of Definition 2.1 of the constrained Cauchy problem [1.1, , 1.3 with initial datum $\rho_{0}^{(i)}$, constraint operator $\mathscr{Q}^{(i)}$ and time horizon $t_{i}-t_{i-1}$.

Then the couple $(\rho, q)$, with $\rho$ defined on $[0, T] \times \mathbb{R}$ by

$$
\rho(t, \cdot)=\rho_{0} \mathbf{1}_{\{0\}}(t)+\sum_{i=1}^{N} \rho^{(i)}\left(t-t_{i-1}, \cdot\right) \mathbf{1}_{\left(t_{i-1}, t_{i}\right]}(t)
$$

\footnotetext{
${ }^{1}$ The constraints $\mathscr{Q}^{(i)}$ depend explicitly on the time variable, through the already constructed functions $\rho^{(j)}, j=1, \ldots, i-1$. This explicit time dependence is handled without any additional difficulty within the construction of Theorem 3.1
} 
and $q$ defined a.e. on $[0, T]$ by

$$
q=\sum_{i=1}^{N} q^{(i)}\left(t-t_{i-1}\right) \mathbf{1}_{\left(t_{i-1}, t_{i}\right]}(t)
$$

is an entropy solution to the constrained Cauchy problem [1.1, [1.3] in the sense of Definition 2.1 with initial datum $\rho_{0}$, constraint operator $\mathscr{Q}$ and time horizon $T$.

Moreover, if at every stage of the construction $\left(\rho^{(i)}, q^{(i)}\right), i=1, \ldots, N$, are unique, then the uniqueness of the entropy solution to [1.1], 1.3) also holds.

Proof. The fact that $(\rho, q)$ given by [3.1), (3.2) is an entropy solution until time $T$ follows by a direct verification of Definition 2.1 globally on $[0, T]$. First, Definition 2.1(ii) comes from the fact that by construction and due to the property $\left[\mathbf{Q}^{\text {hd }}\right.$, we have for all $i=1, \ldots, N$, for a.e. $s \in\left[t_{i-1}, t_{i}\right)$,

$$
\mathscr{Q}[\rho](s)=\mathscr{Q}^{(i)}\left[\rho^{(i)}\right]\left(s+t_{i-1}\right)=q^{(i)}\left(s+t_{i-1}\right)=q(s) .
$$

Second, observe that due to the time continuity of entropy solutions we can extend [2.1a)-(2.1c) to test functions supported in $[0, T] \times \mathbb{R}$ with the additional term $\int_{\mathbb{R}}|\rho(T, x)-k| \phi(T, x) \mathrm{d} x$ in the right-hand side. Therefore it is immediate to check the entropy inequalities for $\rho$ with test functions restricted to $\left[t_{i-1}, t_{i}\right]$; this is a mere time translation of the corresponding inequalities 2.1a)-2.1c] (with the extra term at $t=t_{i}-t_{i-1}$ ) for $\rho^{(i)}$. Then it is enough to sum up the entropy inequalities corresponding to different values of $i$ and to use cancel the terms accounting for $t=t_{i}$, $i=1, \ldots, N-1$, due to the fact that, by construction, $\rho^{(i+1)}(0, \cdot)=\rho^{(i)}\left(t_{i}-t_{i-1}, \cdot\right)$ whence

$$
\int_{\mathbb{R}}\left|\rho^{(i)}\left(t_{i}-t_{i-1}, x\right)-k\right| \phi\left(t_{i}, x\right) \mathrm{d} x-\int_{\mathbb{R}}\left|\rho^{(i+1)}(0, x)-k\right| \phi\left(t_{i}, x\right) \mathrm{d} x=0 .
$$

Finally, assume that at every stage of the construction, uniqueness of $\left(\rho^{(i)}, q^{(i)}\right)$ holds. Assume that $(\widetilde{\rho}, \widetilde{q})$ is a solution on $[0, T]$. The function $\widetilde{\rho}^{(1)}=\left.\widetilde{\rho}\right|_{\left[0, t_{1}\right]}$ solves the same problem as $\rho^{(1)}$, therefore $\widetilde{\rho}^{(1)} \equiv \rho^{(1)}$. Then the initial data and the constraint operators defining $\rho^{(2)}$ and $\widetilde{\rho}^{(2)}:(t, \cdot) \mapsto \rho\left(t+t_{1}, \cdot\right)$ on $\left[0, t_{2}-t_{1}\right]$ coincide, therefore $\widetilde{\rho}^{(2)} \equiv \rho^{(2)}$. Continuing by induction, we find that $\widetilde{\rho}=\rho$ and conclude to uniqueness of the entropy solution to [1.1], [1.3) with initial datum $\rho_{0}$, constraint operator $\mathscr{Q}$ and time horizon $T$.

Before stating our main results, let us recall the uniform Lipschitz continuity estimate for the maps $\mathscr{R}_{t}$ obtained in [6. Proposition 2.10].

Lemma 3.2. For any $q, \widetilde{q} \in \mathbf{L}^{\infty}(0, T)$ and $t \in[0, T]$ we have

$$
\left\|\mathscr{R}_{t}[q]-\mathscr{R}_{t}[\widetilde{q}]\right\|_{\mathbf{C}^{\mathbf{0}}\left(0, t ; \mathbf{L}^{1}\right)} \leq 2\|q-\widetilde{q}\|_{\mathbf{L}^{1}(0, t)} .
$$

Further, let us give constraint-independent bounds in $\mathbf{C}^{\mathbf{0}}\left(0, T ; \mathbf{L}^{\mathbf{1}}\right)$ on the component $\rho$ of entropy solutions of 1.1$]$. They will be used to ensure boundedness and $\mathbf{L}^{1}$-tightness of families of entropy solutions (or approximate entropy solutions) to constrained problems.

Lemma 3.3. Let $\mathbb{T}$ be the triangle $\{(t, x): 0 \leq t \leq T,|x| \leq \operatorname{Lip}(f) t\}$. Fix an initial datum $\rho_{0} \in \mathbf{L}^{\mathbf{1}}\left(\mathbb{R} ;\left[0, \rho_{\max }\right]\right)$ and let $\rho_{\text {free }} \in \mathbf{C}^{\mathbf{0}}\left(0, T ; \mathbf{L}^{\mathbf{1}}\right)$ be the entropy solution of the unconstrained Cauchy problem $[1.1 \mathrm{a}),[1.1 \mathrm{c}$. Then for any constraint $q$, the entropy solution $\rho$ of the constrained Cauchy problem (1.1) verifies

- $\|\rho\|_{\mathbf{C}^{\mathbf{0}\left(0, T ; \mathbf{L}^{\mathbf{1}}\right)}}=\left\|\rho_{0}\right\|_{\mathbf{L}^{\mathbf{1}(\mathbb{R})}} ;$

- $0 \leq \rho \leq \rho_{\max }$ a.e. on $\mathbb{\mathbb { T }}$;

- $\rho \equiv \rho_{\text {free }}$ and $\inf \left(\rho_{0}\right) \leq \rho \leq \sup \left(\rho_{0}\right)$ a.e. on $\mathbb{T}^{c}=([0, T] \times \mathbb{R}) \backslash \mathbb{T}$.

In particular, given $\rho_{0}$ and $T$, the image of $\mathscr{R}_{T}$ is bounded in $\mathbf{C}^{\mathbf{0}}\left(0, T ; \mathbf{L}^{\mathbf{1}}\right)$.

Proof. The first claim is obvious because $\|\rho(t)\|_{\mathbf{L}^{1}(\mathbb{R})}=\left\|\rho_{0}\right\|_{\mathbf{L}^{1}(\mathbb{R})}$ for all $t \geq 0$. Since the speed of propagation of the waves is bounded from above by $\operatorname{Lip}(f)$, the restriction of $\rho$ to $\mathbb{T}^{c}$ can be seen as the entropy solution to a Dirichlet boundary problem defined in a non-cylindrical domain, and then one can observe that the Dirichlet data are ignored according to the Bardos-LeRoux-Nédélec interpretation [10]; cf. [1] for details of the argument. As a consequence, the restriction of $\rho$ to $\mathbb{T}^{c}$ is fully determined by the initial datum alone and thus it coincides with the restriction of $\rho_{\text {free }}$ to $\mathbb{T}^{c}$. The bounds therefore follows readily from the fact that $\rho_{\text {free }}$ satisfies the maximum principle and from the fact that in $\mathbb{T}, \rho$ takes values in $\left[0, \rho_{\max }\right]$. Then the last claim is an immediate consequence of these bounds.

3.2. A well-posedness result. We start with the following well-posedness result.

\section{Theorem 3.1.}

(1) If $\mathscr{Q}$ verifies $\mathbf{Q}^{\mathrm{Lip}}$, then the constrained Cauchy problem (1.1, 1.3) admits one and only one entropy solution in the sense of Definition 2.1

(2) The conclusion of (1) still holds true if $\mathscr{Q}$ is equivalent in the sense of Definition 2.2 to a constraint $\widehat{\mathscr{Q}}$ that verifies the assumption $\mathbf{Q}^{\mathrm{Lip}}$. 
Proof. The second claim obviously follows from the first one. Therefore only the first claim has to be proved. Observe that a couple $(\rho, q) \in \mathbf{C}^{\mathbf{0}}\left(0, T ; \mathbf{L}^{\mathbf{l}}\right) \times \mathbf{L}^{\mathbf{1}}(0, T)$ is an entropy solution of $[1.1)$, (1.3) if and only if $\rho$ is a fixed point of the map $\mathscr{T}_{T}=\mathscr{R}_{T} \circ \mathscr{Q}_{T}$. We will prove the theorem by applying the Banach-Picard fixed point theorem for sufficiently small $T$. The general claim of existence and uniqueness follows then by bootstrapping the construction, according to Lemma3.1

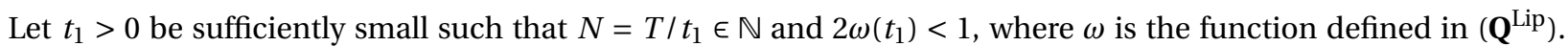
Combining 3.3 with $\mathbf{Q}^{\mathrm{Lip}}$, we find for all $\rho_{1}, \rho_{2} \in \mathbf{C}^{\mathbf{0}}\left(0, T ; \mathbf{L}^{\mathbf{1}}\right)$

$$
\begin{aligned}
& \left\|\mathscr{T}_{t_{1}}\left[\rho_{1}\right]-\mathscr{T}_{t_{1}}\left[\rho_{2}\right]\right\|_{\mathbf{C}^{\mathbf{0}}\left(0, t_{1} ; \mathbf{L}^{\mathbf{1}}\right)} \leq 2\left\|\mathscr{Q}\left[\rho_{1}\right]-\mathscr{Q}\left[\rho_{2}\right]\right\|_{\mathbf{L}^{\mathbf{1}}\left(0, t_{1}\right)} \\
\leq & 2 \omega\left(t_{1}-\tau\right)\left\|\rho_{1}-\rho_{2}\right\|_{\mathbf{C}^{\mathbf{0}}\left(0, t_{1} ; \mathbf{L}^{\mathbf{1}}\right)}<\left\|\rho_{1}-\rho_{2}\right\|_{\mathbf{C}^{\mathbf{0}}\left(0, t_{1} ; \mathbf{L}^{\mathbf{1}}\right)},
\end{aligned}
$$

where $\tau \in\left[0, t_{1}\right]$ is the largest time for which $\rho_{1}(s, \cdot)=\rho_{2}(s, \cdot)$ in $\mathbf{L}^{\mathbf{1}}(\mathbb{R})$ for all $s \in[0, \tau]$. Moreover, according to Lemma 3.3 the image of $\mathscr{R}_{t_{1}}$ is included in a ball $B$ of $\mathbf{C}^{\mathbf{0}}\left(0, t_{1} ; \mathbf{L}^{\mathbf{1}}\right)$ centred in 0 and with radius $\left\|\rho_{0}\right\|_{\mathbf{L}^{\mathbf{1}}(\mathbb{R})}$, therefore, all fixed points of $\mathscr{T}_{t_{1}}$ are included in this ball. Hence we can apply the contractive fixed point theorem to the restriction $\left.\mathscr{T}\right|_{B}$ of $\mathscr{T}$ to $B$ and conclude that there exists a unique solution $\rho^{(1)}$ of our problem with time horizon $t_{1}$. We bootstrap the argument applying Lemma 3.1 with $t_{i}=i t_{1}$. Indeed, using property $\mathbf{Q}^{\text {Lip }}$ with $\tau=t_{1}$ we find the possibility to extend the solution defined on $\left[0, t_{1}\right]$ to the larger interval $\left[0,2 t_{1}\right]$. Continuing in this way, we obtain a uniform partition of $[0, T]$ on which we can apply both existence and uniqueness claims of Lemma 3.1 . This concludes the proof.

In practice, verification of assumption $\mathbf{Q}^{\mathrm{Lip}}$ may be tedious. Therefore we provide the following corollary based on easy-to-check sufficient conditions that express the uniform in $t$ Lipschitz continuity of $\mathscr{Q}_{t}$ with respect to wellchosen couples of norms.

Corollary 3.1. A constraint operator $\mathscr{Q}$ satisfies $\mathbf{Q}^{\text {Lip }}$ and the corresponding constrained Cauchy problem (1.1), (1.3) admits one and only one entropy solution in the sense of Definition 2.1 if there exists a constant $C>0$ such that one of the following conditions is satisfied:

(1) for all $t \in[0, T], \mathscr{Q}_{t}$ verifies

$$
\left\|\mathscr{Q}\left[\rho_{1}\right]-\mathscr{Q}\left[\rho_{2}\right]\right\|_{\mathbf{L}^{\mathbf{1}}(0, t)} \leq C\left\|\rho_{1}-\rho_{2}\right\|_{\mathbf{L}^{\mathbf{1}}\left(0, t ; \mathbf{L}^{\mathbf{1}}\right)} ;
$$

(2) for all $t \in[0, T], \mathscr{Q}_{t}$ verifies

$$
\left\|\mathscr{Q}\left[\rho_{1}\right]-\mathscr{Q}\left[\rho_{2}\right]\right\|_{\mathbf{L}^{\infty}(0, t)} \leq C\left\|\rho_{1}-\rho_{2}\right\|_{\mathbf{C}^{\mathbf{0}}\left(0, t ; \mathbf{L}^{\mathbf{1}}\right)} .
$$

Proof. Assume that $\rho_{1}$ and $\rho_{2}$ coincide in $\mathbf{L}^{\mathbf{1}}(\mathbb{R})$ up to a time $\tau \leq t$.

(1) If $\mathscr{Q}_{t}$ verifies 3.4 for all $t \in[0, T]$, then

$$
\begin{aligned}
\left\|\mathscr{Q}\left[\rho_{1}\right]-\mathscr{Q}\left[\rho_{2}\right]\right\|_{\mathbf{L}^{\mathbf{1}}(0, t)} \leq C\left\|\rho_{1}-\rho_{2}\right\|_{\mathbf{L}^{\mathbf{1}}\left(0, t ; \mathbf{L}^{\mathbf{1}}\right)}= & C \int_{\tau}^{t}\left\|\rho_{1}(s, \cdot)-\rho_{2}(s, \cdot)\right\|_{\mathbf{L}^{\mathbf{1}}(\mathbb{R})} \mathrm{d} s \\
& \leq C(t-\tau) \max _{s \in[\tau, t]}\left\|\rho_{1}(s, \cdot)-\rho_{2}(s, \cdot)\right\|_{\mathbf{L}^{\mathbf{1}}(\mathbb{R})}=C(t-\tau)\left\|\rho_{1}-\rho_{2}\right\|_{\mathbf{C}^{\mathbf{0}}\left(0, t ; \mathbf{L}^{\mathbf{1}}\right)} .
\end{aligned}
$$

(2) First, observe that 3.5 implies property $\mathbf{Q}^{\text {hd }}$. Therefore, $\mathscr{Q}\left[\rho_{1}\right]$ and $\mathscr{Q}\left[\rho_{2}\right]$ also coincide up to the time $\tau$. Hence, if $\mathscr{Q}_{t}$ verifies $[3.5$ for all $t \in[0, T]$, then

$$
\begin{aligned}
\left\|\mathscr{Q}\left[\rho_{1}\right]-\mathscr{Q}\left[\rho_{2}\right]\right\|_{\mathbf{L}^{\mathbf{1}}(0, t)}=\int_{\tau}^{t}\left|\mathscr{Q}\left[\rho_{1}\right](s)-\mathscr{Q}\left[\rho_{2}\right](s)\right| \mathrm{d} s & \leq(t-\tau) \operatorname{ess} \sup _{s \in[\tau, t]}\left|\mathscr{Q}\left[\rho_{1}\right](s)-\mathscr{Q}\left[\rho_{2}\right](s)\right| \\
& =(t-\tau)\left\|\mathscr{Q}\left[\rho_{1}\right]-\mathscr{Q}\left[\rho_{2}\right]\right\|_{\mathbf{L}^{\infty}(0, t)} \leq(t-\tau) C\left\|\rho_{1}-\rho_{2}\right\|_{\mathbf{C}^{\mathbf{0}}\left(0, t ; \mathbf{L}^{\mathbf{1}}\right)} .
\end{aligned}
$$

Thus in both cases we proved that $\mathbf{Q}^{\mathrm{Lip}}$ holds with $\omega=C$ Id and Theorem 3.1 applies.

Remark 3.1. One can also get a result of continuous dependence on $\rho$ using the above technique. To this end, it is enough to include a term in the right-hand side of 3.3 to account for the $\mathbf{L}^{1}$ difference of initial conditions, and to use the version of Banach fixed point theorem with parameter.

However, to state continuous dependence result in a more natural way (in this case, also a dependence on $\mathscr{Q}$ can be studied), one can use the technique of Gronwall inequalities under the appropriate assumptions. We will not pursue this line here, referring to [4 for an example. Observe that continuous dependence estimates obtained with these techniques may be very pessimistic, cf. [2].

3.3. Existence results. While uniqueness and continuous dependence arguments developed or evoked in the previous paragraph seem to require some kind of Lipschitz continuity of $\mathscr{Q}$, existence results can be obtained in much more generality. We have

Theorem 3.2. The constrained Cauchy problem [1.1, 1.3] admits at least one entropy solution in the sense of Definition 2.1 if one of the following conditions is satisfied:

(a) 2 satisfies $\mathbf{Q}^{\mathrm{comp}}$.

(b) $f$ satisfies $\mathbf{G N L}$ and $\mathscr{Q}$ satisfies $\mathbf{Q}^{\text {cont }}$. 
The same conclusion holds if $\mathscr{Q}$ is equivalent to a constraint operator that satisfies (a) or (b).

Proof. As in the proof of Theorem 3.1 we reduce the question of existence of an entropy solution to the problem [1.1, 1.3 to the question of existence of a fixed point for the map $\mathscr{T}_{T}$. Moreover, the last claim is an obvious consequence of the claims of the theorem in the cases (a) and (b), which we now prove.

(a) We have seen in the proof of Theorem 3.1 that the definition of $\mathscr{T}_{T}$ can be restricted to a ball $B$ of $\mathbf{C}^{\mathbf{0}}\left(0, T ; \mathbf{L}^{\mathbf{l}}\right)$ such that $\mathscr{T}_{t}$ acts from the convex set $B$ into itself. Due to estimate 3.3 and assumption $\mathbf{Q}^{\text {comp }}$, the map $\mathscr{T}_{T}$ is continuous from $\mathbf{C}^{\mathbf{0}}\left(0, T ; \mathbf{L}^{\mathbf{1}}\right)$ to itself. Moreover, due to $\mathbf{Q}^{\text {comp }}$ the image $\mathscr{T}_{T}(B)$ is relatively compact in $\mathbf{C}^{\mathbf{0}}\left(0, T ; \mathbf{L}^{\mathbf{1}}\right)$ and one can apply the Schauder fixed point theorem in $\mathbf{C}^{\mathbf{0}}\left(0, T ; \mathbf{L}^{\mathbf{l}}\right)$ to get existence of a solution.

(b) Now, under assumptions $\mathbf{G N L}, \mathbf{Q}^{\text {cont }}$, we will replace the space $\mathbf{C}^{\mathbf{0}}\left(0, T ; \mathbf{L}^{\mathbf{1}}\right)$ by $\mathbf{L}^{\mathbf{1}}\left(0, T ; \mathbf{L}^{\mathbf{1}}\right)$. Then we define $\mathscr{T}_{T}$ from $\mathbf{L}^{\mathbf{1}}\left(0, T ; \mathbf{L}^{\mathbf{1}}\right)$ into $\mathbf{C}^{\mathbf{0}}\left(0, T ; \mathbf{L}^{\mathbf{1}}\right) \subset \mathbf{L}^{\mathbf{1}}\left(0, T ; \mathbf{L}^{\mathbf{1}}\right)$. As before, due to Lemma 3.3 we can restrict this map to the ball $B^{\prime}$ of $\mathbf{L}^{\mathbf{1}}\left(0, T ; \mathbf{L}^{\mathbf{1}}\right)$ centred in 0 and with radius $T\left\|\rho_{0}\right\|_{\mathbf{L}^{\mathbf{1}}(\mathbb{R})}$. We use the continuity assumption $\mathbf{Q}^{\text {cont }}$ along with the estimate 3.3 and the continuous embedding of $\mathbf{C}^{\mathbf{0}}\left(0, T ; \mathbf{L}^{\mathbf{1}}\right)$ into $\mathbf{L}^{\mathbf{1}}\left(0, T ; \mathbf{L}^{\mathbf{1}}\right)$ in order to justify the continuity of this restriction of $\mathscr{T}_{T}$. It remains to prove the relative compactness of the image $\mathscr{T}_{T}\left(B^{\prime}\right)$; then existence of a fixed point of $\mathscr{T}_{t}$ in $\mathscr{T}_{T}\left(B^{\prime}\right) \subset \mathbf{C}^{\mathbf{0}}\left(0, T ; \mathbf{L}^{\mathbf{1}}\right)$ follows, therefore we get an entropy solution to our problem $(1.1$, [1.3). To prove the relative compactness of $\mathscr{T}_{T}\left(B^{\prime}\right)$, observe that one has the $\mathbf{L}_{\mathbf{l o c}}^{\mathbf{l}}((0, T) \times \mathbb{R})$ relative compactness of uniformly bounded families of (local) entropy solutions of equation [1.1a) due to the genuine nonlinearity assumption [GNL (see [45. 43]). It remains to upgrade the $\mathbf{L}_{\text {loc }}^{\mathbf{1}}$ to $\mathbf{L}^{\mathbf{1}}$ compactness, which is possible in our case due to the uniform tightness property for functions in $\mathscr{T}_{T}\left(B^{\prime}\right)$, which is a consequence of the last claim of Lemma 3.3 all functions in $\mathscr{T}_{T}\left(B^{\prime}\right)$ are dominated by a fixed $\mathbf{C}^{\mathbf{0}}\left(0, T ; \mathbf{L}^{\mathbf{l}}\right)$ function $\left.\rho_{\text {free }}\right)$. This concludes the proof of the theorem.

In the spirit of Corollary 3.1 let us point out that the compactness assumption on $\mathscr{Q}$ can follow from the stronger assumption of compactness of $\mathscr{Q}$ as operator from $\mathbf{C}^{\mathbf{0}}\left(0, T ; \mathbf{L}^{\mathbf{1}}\right)$ to $\mathbf{L}^{\infty}(0, T)$; we refer to Section 4 for examples where the latter assumption is easy to check.

\section{BASIC EXAMPLES OF CONSTRAINT OPERATORS}

In this section we introduce some examples of constraint operators satisfying the hypotheses of Theorem 3.1 whence existence and uniqueness results for the corresponding constrained Cauchy problems [1.1, , (1.3) follow. This list is of course not exhaustive, but we indicate several ways to average density or flux values so that to ensure the continuity of averages with respect to the a.e. convergence of solutions. As a consequence it is possible to use these examples as "building blocks" to construct more complex constraint operators to which the theory in the previous section applies.

Example 4.1. Let us consider first the constraint operator $\mathscr{Q}$ defined by (1.5), introduced in the framework of evacuation of a crowd through an exit door in [4] and numerically studied in [3]. Existence and uniqueness of the entropy solution to the constrained Cauchy problem [1.1, [1.3,, 1.5 is proved in [4 Theorem 3.1]. Thanks to Theorem 3.1 we can give a shorter alternative proof which requires weaker hypotheses on $p$ and $w$. However this proof does not lead to a practically implementable construction procedure, and it does not give any hint on the behaviour of the entropy solution nor a priori bounds of its total variation, unlike to the (much longer) arguments of [4]. Observe that in the present setting we do not need to assume GNL for $f$.

First, the property $\mathbf{Q}$ hd is clearly satisfied by (1.5). If $p$ and $w$ are as in $\left(\mathbf{P W K}\right.$, then for any $\rho_{1}, \rho_{2} \in \mathbf{C}^{\mathbf{0}}\left(0, T ; \mathbf{L}^{\mathbf{l}}\right)$ and $t \in[0, T]$ we have

$$
\begin{aligned}
\left\|\mathscr{Q}\left[\rho_{1}\right]-\mathscr{Q}\left[\rho_{2}\right]\right\|_{\mathbf{L}^{\infty}(0, t)} & \leq \operatorname{Lip}(p) \operatorname{ess} \sup _{s \in(0, t)}\left|\int_{\mathbb{R}_{-}}\left[\rho_{1}(s, x)-\rho_{2}(s, x)\right] w(x) \mathrm{d} x\right| \\
& \leq \operatorname{Lip}(p)\|w\|_{\mathbf{L}^{\infty}(\mathbb{R})}\left\|\rho_{1}-\rho_{2}\right\|_{\mathbf{C}^{\mathbf{0}}\left(0, t ; \mathbf{L}^{\mathbf{1}}\right)} .
\end{aligned}
$$

Therefore, condition 3.5 is satisfied with $C=\operatorname{Lip}(p)\|w\|_{\mathbf{L}^{\infty}(\mathbb{R})}$. Hence by virtue of Corollary[3.1 the constraint operator given by (1.5) enjoys $\mathbf{Q}^{\mathrm{Lip}}$ and the constrained Cauchy problem [1.1, [1.3], 1.5] admits one and only one entropy solution in the sense of Definition 2.1

Example 4.2. Consider the case of a constraint operator $\mathscr{Q}[\rho]$ that depends on the values of $\rho$ at times belonging to an a priori fixed discrete set. More specifically, let $t_{1}, \ldots, t_{M} \in[0, T]$ with $t_{i}<t_{i+1}$ and take

$$
\mathscr{Q}[\rho](t)=p(\xi(t)), \quad \xi(t)=\sum_{t_{i} \leq t} \int_{\mathbb{R}} w_{i}(t, x) \rho\left(t_{i}, x\right) \mathrm{d} x,
$$

where $w_{1}, \ldots, w_{M}:[0, T] \times \mathbb{R} \rightarrow \mathbb{R}$ are in $\mathbf{L}^{\infty}$. By definition the property $\left.\mathbf{Q}^{\text {hd }}\right]$ is clearly satisfied. If $p \in \mathbf{L i p}\left(\mathbb{R} ;\left[0, f_{\text {max }}\right]\right)$, then for any $\rho_{1}, \rho_{2} \in \mathbf{C}^{\mathbf{0}}\left(0, T ; \mathbf{L}^{\mathbf{l}}\right)$ and $t \in[0, T]$ we have

$$
\begin{aligned}
\left\|\mathscr{Q}\left[\rho_{1}\right]-\mathscr{Q}\left[\rho_{2}\right]\right\|_{\mathbf{L}^{\infty}(0, t)} & \leq \operatorname{Lip}(p) \operatorname{ess} \sup _{s \in(0, t)}\left|\sum_{t_{i} \leq s} \int_{\mathbb{R}} w_{i}(s, x)\left[\rho_{1}\left(t_{i}, x\right)-\rho_{2}\left(t_{i}, x\right)\right] \mathrm{d} x\right| \\
& \leq\left[\operatorname{Lip}(p) \sum_{i=1}^{M}\left\|w_{i}\right\|_{\mathbf{L}^{\infty}\left(0, T ; \mathbf{L}^{\infty}\right)}\right]\left\|\rho_{1}-\rho_{2}\right\|_{\mathbf{C}^{\mathbf{0}}\left(0, t ; \mathbf{L}^{\mathbf{1}}\right)} .
\end{aligned}
$$


Therefore, condition 3.5 is satisfied with $C=\operatorname{Lip}(p) \sum_{i=1}^{M}\left\|w_{i}\right\|_{\mathbf{L}^{\infty}\left(0, T ; \mathbf{L}^{\infty}\right)}$. Hence, by virtue of Corollary 3.1 the constraint operator given by (4.1) enjoys $\mathbf{Q}^{\mathrm{Lip}}$ and the constrained Cauchy problem [1.1, (1.3), 4.1) admits one and only one entropy solution in the sense of Definition 2.1 .

Example 4.3. Consider a constraint operator $\mathscr{Q}$ such that $\mathscr{Q}[\rho](t)$ depends on the weighted average of $f(\rho)$ in $y \in \mathbb{R}$ on the time interval [max $\{0, t-\tau\}, t]$, with $\tau>0$. This can be expressed by introducing $r \in \mathbf{L i p}\left(\mathbb{R}_{+} ;\left[0, f_{\text {max }}\right]\right), k$ as in $(\overline{\mathbf{P W K}}$ and by requiring that

$$
\mathscr{Q}[\rho](t)=r(\eta(t)), \quad \quad \eta(t)=\int_{0}^{t} \kappa(t-s) f(\rho)\left(s, y^{-}\right) \mathrm{d} s .
$$

Proving any continuity property for $\mathscr{Q}$ is not possible because its definition involves the traces of a function in $\mathbf{L}^{\mathbf{1}}(\mathbb{R} ; \mathbb{R})$; to be precise, the operator $\mathscr{Q}$ is not well defined on the functional spaces we consider. However it is well defined for functions $\rho$ satisfying 1 1.1a) in the weak sense, because the field $(\rho, f(\rho))$ in the $(t, x)$-plane is divergencefree. For this reason we construct a constraint operator $\widehat{\mathscr{Q}}$ equivalent to $\mathscr{Q}$ in the sense of Definition 2.2 which is well defined and for which continuity (and even Lipschitz continuity) can be easily proved. We fix $\phi \in \operatorname{Lip}([0, T] \times(-\infty, y])$ such that $\phi(t, y)=\kappa(t)$ and $x \mapsto \phi(t, x)$ is increasing. Introduce the constraint operator

$$
\widehat{\mathscr{Q}}[\rho](t)=r(\xi(t))
$$

with

$$
\begin{aligned}
\xi & =\xi^{a}+\xi^{b}+\xi^{c}, \\
\xi^{a}(t) & =\int_{0}^{t} \int_{-\infty}^{y}\left[-\rho(s, x) \phi_{t}(t-s, x)+f(\rho)(s, x) \phi_{x}(t-s, x)\right] \mathrm{d} x \mathrm{~d} s, \\
\xi^{b}(t) & =-\int_{-\infty}^{y} \rho(t, x) \phi(0, x) \mathrm{d} x, \\
\xi^{c}(t) & =\int_{-\infty}^{y} \rho_{0}(x) \phi(t, x) \mathrm{d} x .
\end{aligned}
$$

Observe that $\xi^{b}$ and $\xi^{c}$ have the same form as the argument of $p$ in examples 4.1 and 4.2 respectively. The property $\left[\mathbf{Q}^{\text {hd }}\right]$ is clearly satisfied by the constraint operators under consideration. Moreover, by a direct application of the integration by parts formula we obtain the equivalence of the two operators $\mathscr{Q}$ and $\widehat{\mathscr{Q}}$, see Example 2.2

We prove now that $\widehat{\mathscr{Q}}$ satisfies $\mathbf{Q}^{\text {Lip }}$. For any $\rho_{1}, \rho_{2} \in \mathbf{C}^{\mathbf{0}}\left(0, T ; \mathbf{L}^{\mathbf{1}}\right)$ and $t \in[0, T]$ we have

$$
\begin{aligned}
\left|\widehat{\mathscr{Q}}\left[\rho_{1}\right](t)-\widehat{\mathscr{Q}}\left[\rho_{2}\right](t)\right| & \leq \operatorname{Lip}(r)\left[\left|\xi_{1}^{a}(t)-\xi_{2}^{a}(t)\right|+\left|\xi_{1}^{b}(t)-\xi_{2}^{b}(t)\right|\right] \\
& \leq \operatorname{Lip}(r)\left[\operatorname{Lip}(\phi)(1+\operatorname{Lip}(f)) T+\|\phi\|_{\mathbf{C}^{\mathbf{0}}\left(0, t ; \mathbf{L}^{\infty}\right)}\right]\left\|\rho_{1}-\rho_{2}\right\|_{\mathbf{C}^{\mathbf{0}}\left(0, t ; \mathbf{L}^{\mathbf{1}}\right)},
\end{aligned}
$$

where $\xi_{i}^{a}, \xi_{i}^{b}$ correspond to $\rho_{i}, i=1,2$. Therefore $\widehat{\mathscr{Q}}$ satisfies 3.5 and, by Corollary 3.1 it satisfies also $\mathbf{Q}^{\mathrm{Lip}}$ and the constrained Cauchy problem [1.1, (1.3), 4.3) admits one and only one entropy solution in the sense of Definition2.1

Let us underline the following observations:

- In Example $4.2, \xi$ accounts for a space average of the density at some specific moments of time, the value of $\xi$ being transformed into a constraint value by the nonlinear map $p$.

- In Example 4.3 $\eta$ accounts for a time average of the flux at a specific location in space, the value of $\eta$ being transformed into a constraint value by the nonlinear map $r$.

If the flux $f$ is bell shaped, see $\mathbf{F}$, then the link between the two examples for subjective densities lower than $\rho_{c}$ can be provided by the choice $r=p \circ f_{-}^{-1}$, where $f_{-}$is the restriction of $f$ to $\left[0, \rho_{c}\right]$.

Example 4.4. Inspired by the example 2.1 and Section 1.4.3 we propose a class of operators

$$
\widehat{\mathscr{Q}}[\rho](t)=p(\xi(t)),
$$

where $p$ is given by $(\overline{\text { PWK }}$ and $\xi$ is the solution to the Cauchy problem for an ODE

$$
\dot{\xi}(t)=H\left(\xi(t), \int_{\mathbb{R}_{-}} F(t, x, \rho(t, x)) \mathrm{d} x\right), \quad \xi(0)=\xi_{0}\left[\rho_{0}\right] .
$$

Above $\xi_{0}: \mathbf{L}^{\mathbf{1}}(\mathbb{R}) \rightarrow \mathbb{R}$,

$$
H \in \operatorname{Lip}(\mathbb{R} \times \mathbb{R} ; \mathbb{R})
$$

and

$F \in \mathbf{C}_{\mathbf{c}}^{\mathbf{0}}\left([0, T] \times \mathbb{R}_{-} \times\left[0, \rho_{\max }\right] ; \mathbb{R}\right)$ and there exists a constant $L_{F}>0$ such that for any $(t, x) \in[0, T] \times \mathbb{R}_{-}$and $\rho_{1}, \rho_{2} \in\left[0, \rho_{\max }\right]$

$$
\left|F\left(t, x, \rho_{1}\right)-F\left(t, x, \rho_{2}\right)\right| \leq L_{F}\left|\rho_{1}-\rho_{2}\right| .
$$


The property $\widehat{\mathbf{Q}^{\text {hd }}}$ is clearly satisfied by $\widehat{\mathscr{Q}}$. Moreover for any $\rho_{1}, \rho_{2} \in \mathbf{C}^{\mathbf{0}}\left(0, T ; \mathbf{L}^{\mathbf{1}}\right)$ and $t \in[0, T]$, if $\xi_{1}$ and $\xi_{2}$ are the corresponding solutions of 4.5 , then

$$
\begin{aligned}
\left|\dot{\xi}_{1}(t)-\dot{\xi}_{2}(t)\right| & \leq \operatorname{Lip}(H)\left[\left|\xi_{1}(t)-\xi_{2}(t)\right|+\int_{\mathbb{R}_{-}}\left|F\left(t, x, \rho_{1}(t, x)\right)-F\left(t, x, \rho_{2}(t, x)\right)\right| \mathrm{d} x\right] \\
& \leq \operatorname{Lip}(H)\left[\left|\xi_{1}(t)-\xi_{2}(t)\right|+L_{F} \int_{\mathbb{R}_{-}}\left|\rho_{1}(t, x)-\rho_{2}(t, x)\right| \mathrm{d} x\right] \\
& \leq \operatorname{Lip}(H)\left[\left|\xi_{1}(t)-\xi_{2}(t)\right|+L_{F}\left\|\rho_{1}(t, \cdot)-\rho_{2}(t, \cdot)\right\|_{\mathbf{L}^{\mathbf{1}}(\mathbb{R})}\right]
\end{aligned}
$$

and by the Gronwall lemma we have

$$
\begin{aligned}
\left|\xi_{1}(t)-\xi_{2}(t)\right| & \leq \operatorname{Lip}(H) L_{F} \int_{\min \{\tau, t\}}^{t}\left\|\rho_{1}(s, \cdot)-\rho_{2}(s, \cdot)\right\|_{\mathbf{L}^{\mathbf{1}(\mathbb{R})}} e^{\operatorname{Lip}(H)(t-s)} \mathrm{d} s \\
& \leq \operatorname{Lip}(H) L_{F} e^{\operatorname{Lip}(H) T} T\left\|\rho_{1}-\rho_{2}\right\|_{\mathbf{C}^{\mathbf{0}}\left(0, t ; \mathbf{L}^{\mathbf{1}}\right)},
\end{aligned}
$$

where $\tau=\max \left\{s \in[0, T]: \rho_{1}=\rho_{2}\right.$ in $\left.\mathbf{C}^{\mathbf{0}}\left(0, s ; \mathbf{L}^{\mathbf{1}}\right)\right\}$. Therefore, we have that

$$
\left\|\widehat{\mathscr{Q}}_{t}\left[\rho_{1}\right]-\widehat{\mathscr{Q}}_{t}\left[\rho_{2}\right]\right\|_{\mathbf{L}^{\mathbf{1}}(0, t)} \leq\left[\operatorname{Lip}(p) \operatorname{Lip}(H) L_{F} e^{\operatorname{Lip}(H) T} T^{2}\right]\left\|\rho_{1}-\rho_{2}\right\|_{\mathbf{C}^{\mathbf{0}}\left(0, t ; \mathbf{L}^{\mathbf{1}}\right)}
$$

and $\widehat{\mathscr{Q}}_{t}: \mathbf{C}^{\mathbf{0}}\left(0, t ; \mathbf{L}^{\mathbf{1}}\right) \rightarrow \mathbf{L}^{\mathbf{1}}(0, t)$ is Lipschitz continuous. Moreover, we have that

$$
\begin{aligned}
& \left\|\widehat{\mathscr{Q}}_{t}\left[\rho_{1}\right]-\widehat{\mathscr{Q}}_{t}\left[\rho_{2}\right]\right\|_{\mathbf{L}^{\mathbf{1}}(0, t)} \leq \\
\leq & \operatorname{Lip}(p) \operatorname{Lip}(H) L_{F} \int_{0}^{t} \int_{\min \{\tau, s\}}^{s}\left\|\rho_{1}(r, \cdot)-\rho_{2}(r, \cdot)\right\|_{\mathbf{L}^{\mathbf{1}(\mathbb{R})}} e^{\operatorname{Lip}(H)(s-r)} \mathrm{d} r \mathrm{~d} s \\
\leq & {\left[\operatorname{Lip}(p) \operatorname{Lip}(H) L_{F} \int_{0}^{t} \int_{\min \{\tau, s\}}^{s} e^{\operatorname{Lip}(H)(s-r)} \mathrm{d} r \mathrm{~d} s\right]\left\|\rho_{1}-\rho_{2}\right\|_{\mathbf{C}^{\mathbf{0}}\left(0, t ; \mathbf{L}^{\mathbf{1}}\right)} . }
\end{aligned}
$$

It is readily seen that the factor in front of $\left\|\rho_{1}-\rho_{2}\right\|_{\mathbf{C}^{\mathbf{0}}\left(0, t ; \mathbf{L}^{\mathbf{1}}\right)}$ vanishes as $(t-\tau) \rightarrow 0$. In conclusion we proved that $\widehat{\mathscr{Q}}$ satisfies $\mathbf{Q}^{\mathrm{Lip}}$ and the constrained Cauchy problem [1.1], 1.3, (4.4) admits one and only one entropy solution in the sense of Definition 2.1

Remark 4.1. It is easy to relax the hypothesis of Lipschitz continuity on $H$ in Example 4.4 to prove the existence of an entropy solution to [1.1, 1.3, 4.4, 4.5. Indeed equation 4.5 admits a local solution provided the function $H$ is continuous. If in addition $H$ is bounded, then the solution $\xi$ is global and is uniformly in BV. This is enough to ensure that property $\mathbf{Q}^{\text {comp }}$ holds.

Remark 4.2. Observe that the ODE in 2.3 in Example 2.1 can be written in the form 4.5]. Indeed, let $\phi:[0, t] \times \mathbb{R}-\rightarrow$ $\mathbb{R}_{+}$be an arbitrary Lipschitz function such that $\phi(\cdot, 0)=1$ and $\operatorname{supp}(\phi) \subset(0, t) \times \mathbb{R}_{-}$, then by the integration by parts formula and by using the fact that $\rho$ satisfies $\left[1.1 \mathrm{a}\right.$ in $\mathscr{D}^{\prime}([0, T) \times \mathbb{R})$, we can express $\xi$ as follows

$$
\begin{aligned}
\xi(t)-\xi(0) & =\int_{0}^{t}\left\{\int_{\mathbb{R}_{-}} w^{\prime}(x) f(\rho)(s, x) \mathrm{d} x-w(0) f(\rho)\left(s, 0^{-}\right)\right\} \mathrm{d} s \\
& =\int_{0}^{t} \int_{\mathbb{R}_{-}}\left\{w^{\prime}(x) f(\rho)(s, x)-[w(x) \phi(s, x) f(\rho)(s, x)]_{x}\right\} \mathrm{d} x \mathrm{~d} s \\
& =\int_{0}^{t} \int_{\mathbb{R}_{-}}\left\{[w(x)[1-\phi(s, x)]]_{x} f(\rho)(s, x)+w(x) \phi(s, x) \rho_{s}(s, x)\right\} \mathrm{d} x \mathrm{~d} s \\
& =\int_{0}^{t} \int_{\mathbb{R}_{-}}\left\{[w(x)[1-\phi(s, x)]]_{x} f(\rho)(s, x)-w(x) \phi_{s}(s, x) \rho(s, x)\right\} \mathrm{d} x \mathrm{~d} s
\end{aligned}
$$

and we can write the ODE in 2.3 as follows

$$
\dot{\xi}(t)=\int_{\mathbb{R}_{-}}\left\{[w(x)[1-\phi(t, x)]]_{x} f(\rho)(t, x)-w(x) \phi_{t}(t, x) \rho(t, x)\right\} \mathrm{d} x .
$$

Hence 2.3 takes the form 4.5 with $H(\xi, I)=I, \xi_{0}\left[\rho_{0}\right]=\int_{\mathbb{R}_{-}} \rho_{0}(x) \mathrm{d} x$ and

$$
F(t, x, \rho)=[w(x)(1-\phi(t, x))]_{x} f(\rho)-w(x) \phi_{t}(t, x) \rho .
$$

The conditions 4.6, 4.7) are satisfied with $L_{F}=\operatorname{Lip}(w(1-\phi)) \operatorname{Lip}(f)+w(0) \operatorname{Lip}(\phi)$.

In particular the constrained Cauchy problem [1.1, (1.3, 2.2], 2.3) admits one and only one entropy solution in the sense of Definition 2.1

Remark 4.3. With reference to Section 1.4.3, observe that the ODE in 1.8 can be written in the form 4.5. Indeed, analogously to Remark 4.2 for any Lipschitz function $\phi:[0, t] \times \mathbb{R}_{-} \rightarrow \mathbb{R}_{+} \operatorname{such}$ that $\phi(\cdot, 0)=1$ and $\operatorname{supp}(\phi) \subset(0, t) \times \mathbb{R}_{-}$, we can take $H(\xi, I)=\max \{I,-\delta \xi\}$ and

$$
F(t, x, \rho)=[w(x)(1-\phi(t, x))]_{x} f(\rho)-w(x) \phi_{t}(t, x) \rho .
$$


We stress that $F$ is a function; indeed, even if $w$ has a jump at zero, the factor of $w^{\prime}(\cdot)$ is $(1-\phi)$, which makes the product equal to zero at $x=0$. The conditions 4.6, 4.7) are satisfied with $L_{F}=\operatorname{Lip}(w(1-\phi)) \operatorname{Lip}(f)+w(0) \operatorname{Lip}(\phi)$.

In particular the constrained Cauchy problem [1.1, (1.3), 1.7), 1.8) admits one and only one entropy solution in the sense of Definition 2.1.

Observe also that, taking the derivative of the function $\xi$ defined by $4.3 \mathrm{~b}$ and integrating by part $\dot{\xi}_{b}$ defined by (4.3d), we obtain that the constraint operator $\widehat{\mathscr{Q}}$ defined by 4.3 belongs to the class introduced in Example 4.4

\section{Finite VOLUME APPROXIMATION OF NONLOCALLY CONSTRAINED PROBLEMS}

The results in the previous section can be obtained by using a fractional step procedure in the place of fixed point arguments in order to relax the coupling between $\rho$ and $q$. As in the above proofs, Lipschitz continuity estimates or compactness arguments are the cornerstones of the convergence proofs for such approximation procedures. A combination of the classical wave-front tracking algorithm with fractional stepping was used in [4] for the case of the constraint (1.5). The advantage of the wave-front tracking technique is that it is constructive and can be used for numerical simulations (see in particular [4, Section 6]); but, because of the presence of "nonlocal interactions" and of fractional stepping, this algorithm turns out to be very heavy. In this section, we follow the fractional step strategy and the finite volume method to construct convenient numerical approximations. In particular, we design a discretization algorithm for problem [1.1, 1.3) and prove its convergence. The details of the discretization for (1.1) and the scheme are essentially the same as in our work [3] devoted to the particular case of the constraint [1.5]. Here, we focus first on properties required for the discretization of general constraints of the form [1.3], and then we apply the results to several examples of constraints relevant for modelling purposes. The convergence of the scheme will be proved under general assumptions, see $\left[\mathscr{Q}_{\Delta}^{\text {cons }}\right.$ and $\left[\mathscr{Q}_{\Delta}^{\text {comp }}\right.$.

5.1. Non-local constrained finite volume method. In this section we describe the numerical scheme based on finite volume method that we use to solve [1.1, 1.3 and we prove its convergence.

Let $\Delta x$ and $\Delta t$ be the constant space and time steps respectively. Introduce the points $x_{j+1 / 2}=j \Delta x$, the cells $K_{j}=$ $\left[x_{j-1 / 2}, x_{j+1 / 2}\right)$ and the cell centers $x_{j}=(j-1 / 2) \Delta x$ for $j \in \mathbb{Z}$. Let $j_{c}$ be the index such that $x_{j_{c}+1 / 2}$ is the location of the constraint. Define $N=\lfloor T / \Delta t\rfloor$ and for $n \in \mathbb{N} \cap[0, N]$ introduce the time discretization $t^{n}=n \Delta t$. For $n \in \mathbb{N} \cap[0, N]$ and $j \in \mathbb{Z}$, we denote by $\rho_{j}^{n}$ the approximation of the average of $\rho\left(t^{n}, \cdot\right)$ on the cell $K_{j}$, namely

$$
\rho_{j}^{0}=\frac{1}{\Delta x} \int_{K_{j}} \rho_{0}(x) \mathrm{d} x \quad \text { and } \quad \rho_{j}^{n} \simeq \frac{1}{\Delta x} \int_{K_{j}} \rho\left(t^{n}, x\right) \mathrm{d} x \quad \text { if } n>0 .
$$

Let us introduce the discretized initial datum $\rho_{\Delta}^{0}$ defined by

$$
\rho_{\Delta}^{0}(x)=\rho_{j}^{0} \quad \text { for } x \in K_{j} .
$$

If $\rho_{0}$ is a piecewise continuous function, we can re-define $\rho_{j}^{0}$ as point values of $\rho_{0}$ at $x_{j}$ or as the mean of the one-sided limits of $\rho_{0}$ at $x_{j}$ if $x_{j}$ is a point of jump. In all cases, we have convergence of $\rho_{\Delta}^{0}$ to $\rho_{0}$ in $\mathbf{L}^{\mathbf{1}}(\mathbb{R})$, moreover, $\rho_{\Delta}^{0}$ obeys the same $\mathbf{L}^{\infty}$ bounds as $\rho_{0}$. form

We seek for an approximate solution $\left(\rho_{\Delta}, q_{\Delta}\right)$ defined on $[0, T] \times \mathbb{R}$, taking values in $\left[0, \rho_{\max }\right] \times\left[0, f_{\max }\right]$ and of the

$$
\rho_{\Delta}(t, x)=\sum_{n=1}^{N} \rho_{\Delta}^{n}(x) \mathbf{1}_{\left(t^{n-1}, t^{n}\right]}(t), \quad q_{\Delta}(t)=\sum_{n=1}^{N} q_{\Delta}^{n} \mathbf{1}_{\left(t^{n-1}, t^{n}\right]}(t) .
$$

where $\left(\rho_{\Delta}^{n}, q_{\Delta}^{n}\right)_{n=1, \ldots, N}$ are discrete functions computed by executing the following algorithm, based on a suitable discretization $\mathscr{Q}_{\Delta}$ of $\mathscr{Q}$ that satisfies $\left[\mathbf{Q}^{\text {hd }}\right]$.

0 . Initialization. We start with the discretized initial datum $\rho_{\Delta}^{0}$ given in [5.1] and an initial value of the constraint $q_{\Delta}^{0}=\mathscr{Q}_{\Delta}\left[\rho_{\Delta}^{0}\right](0)$.

1. For each $n=0, \ldots, N-1$,

A. We recall that for the unconstrained Cauchy problem [1.1a], [1.1c], a standard finite volume method can be written into the form

$$
\rho_{j}^{n+1}=\rho_{j}^{n}-\frac{\Delta t}{\Delta x}\left(\mathscr{F}_{j+1 / 2}^{n}-\mathscr{F}_{j-1 / 2}^{n}\right),
$$

where $\mathscr{F}_{j+1 / 2}^{n}=F\left(\rho_{j}^{n}, \rho_{j+1}^{n}\right)$ is a monotone, consistent numerical flux, that is, $F$ satisfies the following assumptions:

* $F$ is Lipschitz continuous from $\left[0, \rho_{\max }\right]^{2}$ to $\mathbb{R}$ with Lipschitz constant $\operatorname{Lip}(F)$,

* $F(a, a)=f(a)$ for any $a \in\left[0, \rho_{\max }\right]$,

* $\left[0, \rho_{\max }\right]^{2} \ni(a, b) \mapsto F(a, b) \in\left[0, f_{\max }\right]$ is non-decreasing with respect to $a$ and non-increasing with respect to $b$. 
We also recall that in [6] the numerical flux for the Cauchy problem [1.1 with time dependent constraint $q$ is modified as follows in order to take into account the constraint condition

$$
\mathscr{F}_{j+1 / 2}^{n}= \begin{cases}F\left(\rho_{j}^{n}, \rho_{j+1}^{n}\right) & \text { if } j \neq j_{c}, \\ \min \left\{F\left(\rho_{j}^{n}, \rho_{j+1}^{n}\right), q_{\Delta}^{n}\right\} & \text { if } j=j_{c},\end{cases}
$$

where $q_{\Delta}^{n}$ is an approximation of $q\left(t^{n}\right)$.

B. Given $\left(\rho_{\Delta}^{k}\right)_{k=1, \ldots, n}$ with

$$
\rho_{\Delta}^{n}(x)=\rho_{j}^{n} \quad \text { for } x \in K_{j},
$$

we compute $q_{\Delta}^{n} \in\left[0, f_{\max }\right]$ by discretizing relation $[1.3$ :

$$
q_{\Delta}^{n}=\mathscr{Q}_{\Delta}\left[\mathscr{E}^{n}\left[\rho_{\Delta}^{n}\right]\right]\left(t^{n}\right)
$$

where

$$
\mathscr{E}^{n}\left[\rho_{\Delta}^{n}\right](t, x)=\sum_{k=1}^{n} \rho_{\Delta}^{k}\left(t-t^{k-1}, x\right) \mathbf{1}_{\left(t^{k-1}, t^{k}\right]}(t) .
$$

In the sequel, we will operate with the norm $\|\cdot\|_{\mathbf{L}^{\mathbf{1}}(0, T)}$ for $q_{\Delta}$ and with the norm $\|\cdot\|_{\mathbf{L}^{\infty}\left(0, T ; \mathbf{L}^{1}\right)}$ for $\rho_{\Delta}$, both norms being computed from the above expressions of $q_{\Delta}$ and $\rho_{\Delta}$ as functions of $t$ and $(t, x)$, respectively.

To prove convergence for this algorithm, we will need two specific properties of the discrete constraint operator $\mathscr{Q}_{\Delta}$. First, we assume the following consistency for the approximation of $\mathscr{Q}$ by $\mathscr{Q}_{\Delta}$ :

$\left(\mathscr{Q}_{\Delta}^{\text {cons }}\right)$

$$
\left[q_{\Delta}=\mathscr{Q}_{\Delta}\left[\rho_{\Delta}\right],\left\|q_{\Delta}-q\right\|_{\mathbf{L}^{\mathbf{1}}(0, T)} \rightarrow 0 \text { and }\left\|\rho_{\Delta}-\rho\right\|_{\mathbf{L}^{\mathbf{1}}\left(0, T ; \mathbf{L}^{\mathbf{1}}\right)} \rightarrow 0\right] \Longrightarrow q=\mathscr{Q}[\rho] .
$$

Next, we require an asymptotic compactness property which we expect to be compatible with constraints satisfying the compactness assumption $\mathbf{Q}^{\text {comp }}$, but also with some other constraints like (1.2) thanks to the idea of equivalence between constraints introduced in Definition 2.2

$$
\left(\mathscr{Q}_{\Delta}^{\text {comp }}\right) \quad\left[\begin{array}{l}
\text { the sequence of approximate solutions }\left(\rho_{\Delta}\right) \text { obtained } \\
\text { with the above algorithm is bounded in } \mathbf{L}^{\infty}\left(0, T ; \mathbf{L}^{\mathbf{1}}\right) \\
\text { and } q_{\Delta}=\mathscr{Q}_{\Delta}\left[\rho_{\Delta}\right]
\end{array}\right] \Longrightarrow\left(q_{\Delta}\right) \text { is compact in } \mathbf{L}^{\mathbf{1}}(0, T) \text {. }
$$

Here and in the sequel, by compactness of $\left(q_{\Delta}\right)$ we mean the possibility to extract a convergent subsequence in $\mathbf{L}^{\mathbf{1}}(0, T)$, i.e., the relative compactness.

Remark 5.1. If a given constraint operator $\mathscr{Q}$ verifies $\mathbf{Q}^{\text {comp }}$, it is natural to construct discrete constraint operators $\left(\mathscr{Q}_{\Delta}\right)$ which verify $\mathscr{Q}_{\Delta}^{\text {comp }}$ irrespective of the nature of the sequence $\left(\rho_{\Delta}\right)$.

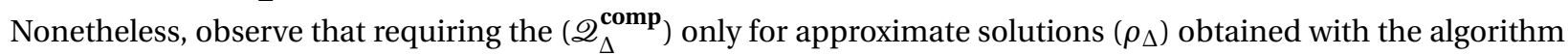
described in this section makes the property much weaker: the sequence $\left(\rho_{\Delta}\right)$ need not to be arbitrary, but it solves

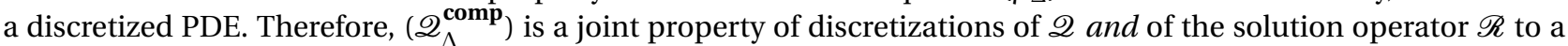
constrained Cauchy problem for a conservation law.

In this section, we do not formalize the idea of equivalence of two discrete constraint operators $\mathscr{Q}_{\Delta}$ and $\widehat{\mathscr{Q}}_{\Delta}$ like this was done in Definition 2.2 for the continuous case; but in the examples, we exploit the same idea by proving property $\mathscr{Q}_{\Delta}^{\text {comp }}$ due to a transformation of $\mathscr{Q}_{\Delta}$ into a more convenient form, using the "weak formulation" of the scheme [5.2].

The alternative line of analysis, with space $\mathbf{C}^{\mathbf{0}}\left(0, T ; \mathbf{L}^{\mathbf{1}}\right)$ for $\rho$ replaced by $\mathbf{L}^{\mathbf{1}}\left(0, T ; \mathbf{L}^{\mathbf{1}}\right)$ and asymptotic compactness for $\mathscr{Q}_{\Delta}$ replaced by the genuine nonlinearity assumption $\mathbf{G N L}$ and a kind of asymptotic continuity assumption on $\left(\mathscr{Q}_{\Delta}\right)$ is briefly discussed in Remark 5.2

5.2. Convergence of the scheme. Let us notice that as in [6. Proposition 4.2], under the CFL condition

$$
\operatorname{Lip}(F) \frac{\Delta t}{\Delta x} \leq \frac{1}{2},
$$

we have the $\mathbf{L}^{\infty}$ stability of the scheme [5.2], [5.3), [5.4), that is

$$
0 \leq \rho_{\Delta}(t, x) \leq \rho_{\max }
$$

for a.e. $(t, x) \in(0, T) \times \mathbb{R}$

We are now in a position to prove a convergence result for the scheme [5.2), [5.3, [5.4). We have the following results

Theorem 5.1. Let $\mathscr{Q}$ verify $\mathbf{Q}^{\mathrm{Lip}}$ and $(\widetilde{\rho}, \widetilde{q})$ be the unique entropy solution of (1.1), 1.3 in the sense of Definition 2.1 .

(1) Assume that the approximation $\mathscr{Q}_{\Delta}$ of $\mathscr{Q}$ is consistent in the sense $\left[\mathscr{Q}_{\Delta}^{\text {cons }}\right.$ and asymptotically compact in the sense $\mathscr{Q}_{\Delta}^{\text {comp. }}$. Then $\left(\rho_{\Delta}, q_{\Delta}\right)$ constructed by the scheme [5.2], [5.3, [5.4] converges to $(\tilde{\rho}, \tilde{q})$ as $\Delta t, \Delta x \rightarrow 0$.

(2) If $\mathscr{Q}$ and $\widehat{\mathscr{Q}}$ are equivalent in the sense of Definition 2.2 and $\widehat{\mathscr{Q}}$ admits an approximation $\widehat{\mathscr{Q}}_{\Delta}$ that satisfies $\mathscr{Q}_{\Delta}^{\text {cons }}$ and $\mathscr{Q}_{\Delta}^{\text {comp }}$, then $\left(\widehat{\rho}_{\Delta}, \widehat{q}_{\Delta}\right)$ constructed by the scheme (5.2), [5.3, (5.4) corresponding to $\widehat{\mathscr{Q}}_{\Delta}$ converges to $(\tilde{\rho}, \tilde{q})$ as $\Delta t, \Delta x \rightarrow 0$. 
Proof. (1) By construction we have that $\left(\rho_{\Delta}\right)$ is uniformly bounded in $\mathbf{L}^{\infty}\left(0, T ; \mathbf{L}^{\mathbf{l}}\right)$. Therefore $\mathscr{Q}_{\Delta}^{\text {comp }}$ gives the existence of a subsequence of $\left(q_{\Delta}\right)$, still denoted $\left(q_{\Delta}\right)$ and a constraint function $q \in \mathbf{L}^{\mathbf{1}}(0, T)$ such that $\left(q_{\Delta}\right)$ converges to $q$ strongly in $\mathbf{L}^{\mathbf{1}}(0, T)$ as $\Delta t \rightarrow 0$. Let $\rho \in \mathbf{C}^{\mathbf{0}}\left([0, T] ; \mathbf{L}_{\text {loc }}^{\mathbf{1}}\left(\mathbb{R} ;\left[0, \rho_{\max }\right]\right)\right)$ be the unique entropy solution to (1.1) in the sense of Definition 2.1 (i) associated to $q$. We first prove that the subsequence $\left(\rho_{\Delta}\right)$ converges to $\rho$ strongly in $\mathbf{L}^{\mathbf{1}}\left(0, T ; \mathbf{L}^{\mathbf{l}}\right)$ as $\Delta t, \Delta x \rightarrow 0$. Let $\widehat{q}_{\Delta}$ be a piecewise constant (with step $\Delta t$ ) approximation of $q$ such that $\widehat{q}_{\Delta}$ converges to $q$ strongly in $\mathbf{L}^{\mathbf{1}}(0, T)$. Let us introduce the auxiliary sequence $\widehat{\rho}_{\Delta}$ constructed by the scheme [5.2], [5.3) and associated to the sequence of discrete constraints $\widehat{q}_{\Delta}$. We have

$$
\left\|\rho-\rho_{\Delta}\right\|_{\mathbf{L}^{\mathbf{1}}\left(0, T ; \mathbf{L}^{\mathbf{1}}\right)} \leq\left\|\rho-\widehat{\rho}_{\Delta}\right\|_{\mathbf{L}^{\mathbf{1}}\left(0, T ; \mathbf{L}^{\mathbf{1}}\right)}+\left\|\rho_{\Delta}-\widehat{\rho}_{\Delta}\right\|_{\mathbf{L}^{\mathbf{1}}\left(0, T ; \mathbf{L}^{\mathbf{1}}\right)} .
$$

But, thanks to [6. Theorem 4.9], under the CFL condition [5.5], $\left\|\rho-\widehat{\rho}_{\Delta}\right\|_{\mathbf{L}^{\mathbf{1}}\left(0, T ; \mathbf{L}^{\mathbf{1}}\right)}$ tends to 0 as $\Delta t, \Delta x \rightarrow 0$. Furthermore, thanks to [3. Proposition 3.1], we have

$$
\left\|\rho_{\Delta}-\widehat{\rho}_{\Delta}\right\|_{\mathbf{L}^{\mathbf{1}}\left(0, T ; \mathbf{L}^{\mathbf{1}}\right)} \leq 2 T\left\|q_{\Delta}-\widehat{q}_{\Delta}\right\|_{\mathbf{L}^{\mathbf{1}}(0, T)},
$$

which also shows that $\left\|\rho_{\Delta}-\widehat{\rho}_{\Delta}\right\|_{\mathbf{L}^{1}\left(0, T ; \mathbf{L}^{1}\right)}$ tends to 0 as $\Delta t, \Delta x \rightarrow 0$. Now, by the definition of $\rho$, the couple $(\rho, q)$ fulfills Definition 2.1 (i). Moreover, due to the above convergences of $\left(\rho_{\Delta}\right)$ and $\left(q_{\Delta}\right)$ and the consistency property $\left.\mathscr{Q}_{\Delta}^{\text {cons }}\right]$, it follows that $(1.3)$ holds. Thus $(\rho, q)$ coincides with the unique entropy solution $(\tilde{\rho}, \tilde{q})$. Finally, it is classical that the extraction of a subsequence can be bypassed due to the uniqueness of $(\tilde{\rho}, \tilde{q})$.

(2) This item follows from the definition of equivalent operators Definition 2.2 and (1).

Remark 5.2. We think that, like for the existence proof, in the above convergence result the discrete compactness assumption $\mathbf{Q}^{\text {comp }}$ can be replaced by a discrete analogous of the mere continuity property $\mathbf{Q}^{\text {cont }}$, whenever the genuine nonlinearity assumption $\mathbf{G N L}$ on $f$ is imposed. In this case, contrarily to the above proof one has to ensure in the first step the relative compactness of $\left(\rho_{\Delta}\right)$. In the second step, discrete analogous of $\mathbf{Q}^{\text {cont }}$ will ensure convergence of the sequence $\left(q_{\Delta}\right)$ associated to the convergent subsequence of $\left(\rho_{\Delta}\right)$. Finally, $\mathscr{Q}_{\Delta}^{\text {cons }}$ will permit us to link the respective limits $\rho$ and $q$ by the constraint relation [1.3].

However, quite technical arguments of the compensated compactness kind, based on GNL, are required in order to handle $\left(\rho_{\Delta}\right)$ at the first step. Therefore we limit our convergence study to the setting of Theorem 5.1 Notice that such alternative kind of compactness argument is however needed in studying approximations of the constrainted Aw, Rascle [9] and Zhang [52] model studied in [5], since the arguments in [6. Theorem 4.9] and in [3] Proposition 3.1] are specific to the scalar case. Studies of models as in [5] and of their approximation are postponed to future work.

\section{EXAMPLES OF DISCRETE CONSTRAINT OPERATORS}

In this section we apply the theory developed in Section 5 to construct and study discrete constraint operators corresponding to the constraint operators introduced in Section 4 . In particular we prove that the hypotheses of Theorem 5.1 are satisfied and we infer the convergence of the scheme [5.2], [5.3), [5.4] as $\Delta t, \Delta x \rightarrow 0$ to the unique entropy solution of the corresponding constrained Cauchy problem [1.1, [1.3) in the sense of Definition 2.1.

Example 6.1. With reference to [4], we recall that as a discrete version of the constraint operator $\mathscr{Q}$ defined in $[1.5]$, in [3] the authors introduce

$$
\mathscr{Q}_{\Delta}\left[\rho_{\Delta}\right](t)=p\left(\Delta x \sum_{j=j_{w}}^{j_{c}} \rho_{j}^{n} w_{j}\right) \quad \text { for } t \in\left(t^{n-1}, t^{n}\right],
$$

where $w_{j}=\frac{1}{\Delta x} \int_{K_{j}} w(x) \mathrm{d} x$ and $j_{w}$ is an integer such that $\operatorname{supp}(w) \subset \bigcup_{j_{w} \leq j \leq j_{c}} K_{j}$. Let's prove first that $\mathscr{Q}_{\Delta}$ enjoys property $\mathscr{Q}_{\Delta}^{\text {cons }}$. If $\left\|\mathscr{Q}_{\Delta}\left[\rho_{\Delta}\right]-q\right\|_{\mathbf{L}^{1}(0, T)} \rightarrow 0$ and $\left\|\rho_{\Delta}-\rho\right\|_{\mathbf{L}^{1}\left(0, T ; \mathbf{L}^{\mathbf{1}}\right)} \rightarrow 0$, then

$$
\begin{aligned}
& \left\|\mathscr{Q}[\rho]-\mathscr{Q}_{\Delta}\left[\rho_{\Delta}\right]\right\|_{\mathbf{L}^{\mathbf{1}}(0, T)} \leq \operatorname{Lip}(p) \sum_{j=j_{w}}^{j_{c}}\left\|\int_{K_{j}}\left[\rho(\cdot, x) w(x)-\rho_{\Delta}(\cdot, x) w_{j}\right] \mathrm{d} x\right\|_{\mathbf{L}^{\mathbf{1}}(0, T)} \\
\leq & \operatorname{Lip}(p) \sum_{j=j_{w}}^{j_{c}}\left[\left\|\int_{K_{j}} \rho(\cdot, x)\left[w(x)-w_{j}\right] \mathrm{d} x\right\|_{\mathbf{L}^{\mathbf{1}}(0, T)}+\left\|\int_{K_{j}}\left[\rho(\cdot, x)-\rho_{\Delta}(\cdot, x)\right] w_{j} \mathrm{~d} x\right\|_{\mathbf{L}^{\mathbf{1}}(0, T)}\right] \\
\leq & \operatorname{Lip}(p) \sum_{j=j_{w}}^{j_{c}}\left[\rho_{\max } T \int_{K_{j}}\left|w(x)-w_{j}\right| \mathrm{d} x+\|w\|_{\mathbf{L}^{\infty}(\mathbb{R})}\left\|\int_{K_{j}}\left|\rho(\cdot, x)-\rho_{\Delta}(\cdot, x)\right| \mathrm{d} x\right\|_{\mathbf{L}^{\mathbf{1}}(0, T)}\right] \\
\leq & \operatorname{Lip}(p)\left[\rho_{\max } T \sum_{j=j_{w}}^{j_{c}} \int_{K_{j}}\left|w(x)-w_{j}\right| \mathrm{d} x+\|w\|_{\mathbf{L}^{\infty}(\mathbb{R})}\left\|\rho-\rho_{\Delta}\right\|_{\mathbf{L}^{\mathbf{1}}\left(0, T ; \mathbf{L}^{\mathbf{1}}\right)}\right] \underset{\Delta x \rightarrow 0}{\longrightarrow} 0 .
\end{aligned}
$$

Finally, let us prove also that $\mathscr{Q}_{\Delta}^{\text {comp }}$ holds. By definition $\left(\rho_{\Delta}\right)$ are constructed by applying a conservative finite volume method, hence

$$
\left\|\rho_{\Delta}\right\|_{\mathbf{L}^{\infty}\left(0, T ; \mathbf{L}^{\mathbf{l}}\right)}=\left\|\rho_{\Delta}^{0}\right\|_{\mathbf{L}^{\mathbf{1}(\mathbb{R})}}=\left\|\rho_{0}\right\|_{\mathbf{L}^{\mathbf{1}(\mathbb{R})}} .
$$


We need to justify that $\left(\mathscr{Q}_{\Delta}\left[\rho_{\Delta}\right]\right)$ is compact in $\mathbf{L}^{\mathbf{1}}(0, T)$. This can be done by changing $\mathscr{Q}_{\Delta}$ under an equivalent form, see the proof of [3. Proposition 3.2], under the additional assumption of Lipschitz continuity of $w$. Namely, by [3. Proposition 3.2] we have that $\left(\mathscr{Q}_{\Delta}\left[\rho_{\Delta}\right]\right)$ is uniformly bounded in $\mathbf{B V}$, and therefore it is compact in $\mathbf{L}^{\mathbf{l}}(0, T)$. In conclusion, Theorem 5.1 applies if $w$ satisfies wLip.

Example 6.2. With reference to Example 4.2 let us introduce as a discrete version of operator defined in 4.1] the discrete constraint operator

$$
\mathscr{Q}_{\Delta}\left[\rho_{\Delta}\right](t)=p\left(\xi_{\Delta}\right), \quad \xi_{\Delta}=\sum_{t_{i}<t} \int_{\mathbb{R}} w_{i}^{\Delta}(t, x) \rho_{\Delta}\left(t_{i}, x\right) \mathrm{d} x
$$

where

$$
w_{i}^{\Delta}(t, x)=\frac{1}{\Delta x} \int_{K_{j}} w_{i}\left(t^{n-1}, y\right) \mathrm{d} y, \quad \text { for } t \in\left(t^{n-1}, t^{n}\right] \text { and } x \in K_{j} .
$$

For simplicity assume that

$$
\begin{aligned}
& \text { for any } t \in[0, T] \text { we have } x \mapsto w_{i}(t, x) \text { is in } \operatorname{Lip}(\mathbb{R} ; \mathbb{R}) \text { and } \\
& \text { for any } x \in \mathbb{R} \text { we have } t \mapsto w_{i}(t, x) \text { is piecewise constant. }
\end{aligned}
$$

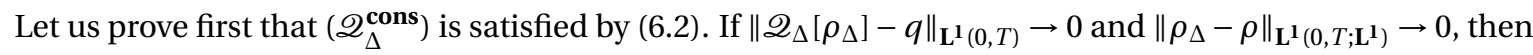

$$
\begin{aligned}
& \left\|\mathscr{Q}[\rho]-\mathscr{Q}_{\Delta}\left[\rho_{\Delta}\right]\right\|_{\mathbf{L}^{\mathbf{1}}(0, T)} \leq \operatorname{Lip}(p) \int_{0}^{T}\left|\sum_{t_{i} \leq t} \int_{\mathbb{R}}\left[w_{i}(t, x) \rho\left(t_{i}, x\right)-w_{i}^{\Delta}(t, x) \rho_{\Delta}\left(t_{i}, x\right)\right] \mathrm{d} x\right| \mathrm{d} t \\
\leq & \operatorname{Lip}(p) \int_{0}^{T} \sum_{t_{i} \leq t} \int_{\mathbb{R}}\left[\left|w_{i}(t, x)-w_{i}^{\Delta}(t, x)\right| \rho\left(t_{i}, x\right)+w_{i}^{\Delta}(t, x)\left|\rho\left(t_{i}, x\right)-\rho_{\Delta}\left(t_{i}, x\right)\right|\right] \mathrm{d} x \mathrm{~d} t \\
\leq & \operatorname{Lip}(p) \sum_{i=1}^{M}\left[\left\|w_{i}-w_{i}^{\Delta}\right\|_{\mathbf{L}^{\mathbf{1}}\left(0, T ; \mathbf{L}^{\mathbf{1}}\right)} \rho_{\max }+\left\|w_{i}\right\|_{\mathbf{L}^{\mathbf{1}}\left(0, T ; \mathbf{L}^{\infty}\right)}\left\|\rho-\rho_{\Delta}\right\|_{\mathbf{L}^{\mathbf{1}}\left(0, T ; \mathbf{L}^{\mathbf{1}}\right)}\right] \underset{\Delta x \rightarrow 0}{\longrightarrow} 0 .
\end{aligned}
$$

Finally, it is immediate to check the compactness property $\mathscr{Q}_{\Delta}^{\text {comp }}$ because $p$ is continuous and the sequence $\left(\xi_{\Delta}\right)$ takes values in a finite dimensional subset of $\mathbf{L}^{\mathbf{1}}(0, T)$ due to assumption (wLipPC).

Example 6.3. With reference to Example 4.3 let $j_{s}$ be the index such that $x_{j_{s}+1 / 2}$ is the location of $y$. We introduce as a discrete version of the constraint operator defined in 4.2 the discrete constraint operator

$$
\mathscr{Q}_{\Delta}\left[\rho_{\Delta}\right]=r\left(\eta_{\Delta}\right)
$$

where

$$
\begin{array}{ll}
\eta_{\Delta}(t)=\Delta t \sum_{m=0}^{n} \kappa^{n-m} \mathscr{F}_{j_{s}+1 / 2}^{m} & \text { for } t \in\left(t^{n}, t^{n+1}\right] \\
\kappa^{0}=\kappa(0) & \\
\kappa^{m+1}=\frac{1}{\Delta t} \int_{t^{m}}^{t^{m+1}} \kappa(s) \mathrm{d} s . &
\end{array}
$$

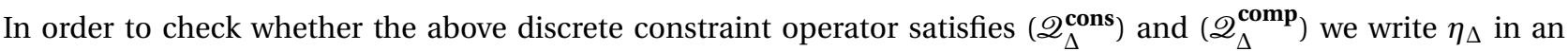
equivalent form. Let $\left(\phi_{j}^{m}\right)$ be a discretization of $\phi$ such that $\phi_{j_{s}}^{m}=\kappa^{m}$. Since $\phi$ is Lipschitz continuous we have that there exists a constant $C>0$ such that

Observe that

$$
\left|\phi_{j}^{m+1}-\phi_{j}^{m}\right| \leq C \Delta t, \quad\left|\phi_{j+1}^{m}-\phi_{j}^{m}\right| \leq C \Delta x .
$$

$$
\begin{aligned}
& \sum_{m=0}^{n}\left(\rho_{j}^{m+1}-\rho_{j}^{m}\right) \phi_{j}^{n-m}=\rho_{j}^{n+1} \phi_{j}^{0}-\rho_{j}^{0} \phi_{j}^{n+1}+\sum_{m=0}^{n} \rho_{j}^{m}\left(\phi_{j}^{n+1-m}-\phi_{j}^{n-m}\right), \\
& \sum_{j \leq j_{s}}\left(\mathscr{F}_{j+1 / 2}^{m}-\mathscr{F}_{j-1 / 2}^{m}\right) \phi_{j}^{n-m}=\mathscr{F}_{j_{s}+1 / 2}^{m} \phi_{j_{s}}^{n-m}-\sum_{j \leq j_{s}} \mathscr{F}_{j-1 / 2}^{m}\left(\phi_{j}^{n-m}-\phi_{j-1}^{n-m}\right) .
\end{aligned}
$$

Therefore, multiplying by $\phi_{j}^{n-m}$ the equality

$$
\frac{\rho_{j}^{m+1}-\rho_{j}^{m}}{\Delta t}=-\frac{\mathscr{F}_{j+1 / 2}^{m}-\mathscr{F}_{j-1 / 2}^{m}}{\Delta x}
$$

derived from (5.2) and adding over $j \leq j_{s}$ and $m \leq n$ give

$$
\begin{aligned}
& \frac{1}{\Delta t} \sum_{j \leq j_{s}}\left[\rho_{j}^{n+1} \phi_{j}^{0}-\rho_{j}^{0} \phi_{j}^{n+1}+\sum_{m=0}^{n} \rho_{j}^{m}\left(\phi_{j}^{n+1-m}-\phi_{j}^{n-m}\right)\right] \\
= & -\frac{1}{\Delta x} \sum_{m=0}^{n}\left[\mathscr{F}_{j_{s}+1 / 2}^{m} \phi_{j_{s}}^{n-m}-\sum_{j \leq j_{s}} \mathscr{F}_{j-1 / 2}^{m}\left(\phi_{j}^{n-m}-\phi_{j-1}^{n-m}\right)\right] .
\end{aligned}
$$


Thus for any $t \in\left(t^{n}, t^{n+1}\right]$ we have that

$$
\begin{array}{r}
\eta_{\Delta}(t)=\Delta t \sum_{m=0}^{n} \kappa^{n-m} \mathscr{F}_{j_{s}+1 / 2}^{m}=\Delta t \sum_{m=0}^{n} \phi_{j_{s}}^{n-m} \mathscr{F}_{j_{s}+1 / 2}^{m}=\Delta x \sum_{j \leq j_{s}}\left[\rho_{j}^{0} \phi_{j}^{n+1}-\rho_{j}^{n+1} \phi_{j}^{0}\right] \\
+\Delta t \Delta x \sum_{m=0}^{n} \sum_{j \leq j_{s}}\left[-\rho_{j}^{m} \frac{\phi_{j}^{n+1-m}-\phi_{j}^{n-m}}{\Delta t}+\mathscr{F}_{j-1 / 2}^{m} \frac{\phi_{j}^{n-m}-\phi_{j-1}^{n-m}}{\Delta x}\right] .
\end{array}
$$

Observe that in this way we obtained a discretization $\widehat{\mathscr{Q}}_{\Delta}$ of the operator $\widehat{\mathscr{Q}}$ appearing in 4.3 . Recall also that the definition 4.2 of $\mathscr{Q}$ is a formal definition, while $\widehat{\mathscr{Q}}$ in 4.3 is rigorous. Therefore it is natural to prove $\mathscr{Q}_{\Delta}^{\text {cons }}$ for the discretized constraint operator by comparing it to $\widehat{\mathscr{Q}}$. For simplicity, assume $j_{c} \neq j_{s}$. Let $f(\rho)(t, \cdot)=0$ for all $t<0$ and $\mathscr{F}^{h}=0$ for all $h<0$. Assume that $\left\|\widehat{\mathscr{Q}}_{\Delta}\left[\rho_{\Delta}\right]-q\right\|_{\mathbf{L}^{\mathbf{1}(0, T)}} \rightarrow 0$ and $\left\|\rho_{\Delta}-\rho\right\|_{\mathbf{L}^{\mathbf{1}}\left(0, T ; \mathbf{L}^{\mathbf{1}}\right)} \rightarrow 0$, then

$$
\begin{aligned}
& \left\|\widehat{\mathscr{Q}}[\rho]-\mathscr{Q}_{\Delta}\left[\rho_{\Delta}\right]\right\|_{\mathbf{L}^{1}(0, T)} \leq \\
& \leq \operatorname{Lip}(r) \sum_{n=0}^{N-1} \int_{t^{n}}^{t^{n+1}}\left|\xi^{a}(t)-\Delta t \Delta x \sum_{m=0}^{n} \sum_{j \leq j_{s}}\left[-\rho_{j}^{m} \frac{\phi_{j}^{n+1-m}-\phi_{j}^{n-m}}{\Delta t}+\mathscr{F}_{j-1 / 2}^{m} \frac{\phi_{j}^{n-m}-\phi_{j-1}^{n-m}}{\Delta x}\right]\right| \mathrm{d} t \\
& \quad+\operatorname{Lip}(r) \sum_{n=0}^{N-1} \int_{t^{n}}^{t^{n+1}}\left|\xi^{b}(t)+\Delta x \sum_{j \leq j_{s}} \rho_{j}^{n+1} \phi_{j}^{0}\right|+\operatorname{Lip}(r) \sum_{n=0}^{N-1} \int_{t^{n}}^{t^{n+1}}\left|\xi^{c}(t)-\Delta x \sum_{j \leq j_{s}} \rho_{j}^{0} \phi_{j}^{n+1}\right| \mathrm{d} t \underset{\Delta x \rightarrow 0}{\longrightarrow} 0 .
\end{aligned}
$$

Indeed we have that

$$
\begin{aligned}
& \left|\xi^{a}(t)-\Delta t \Delta x \sum_{m=0}^{n} \sum_{j \leq j_{s}}\left[-\rho_{j}^{m} \frac{\phi_{j}^{n+1-m}-\phi_{j}^{n-m}}{\Delta t}+\mathscr{F}_{j-1 / 2}^{m} \frac{\phi_{j}^{n-m}-\phi_{j-1}^{n-m}}{\Delta x}\right]\right| \\
& \leq \sum_{m=0}^{n} \sum_{j \leq j_{s}} \int_{t^{m}}^{t^{m+1}} \int_{K_{j}}\left|-\rho(s, x) \phi_{t}(t-s, x)+\rho_{j}^{m} \frac{\phi_{j}^{n+1-m}-\phi_{j}^{n-m}}{\Delta t}\right| \mathrm{d} x \mathrm{~d} s \\
& \quad+\sum_{m=0}^{n} \sum_{j \leq j_{s}} \int_{t^{m}}^{t^{m+1}} \int_{K_{j}}\left|f(\rho)(s, x) \phi_{x}(t-s, x)-\mathscr{F}_{j-1 / 2}^{m} \frac{\phi_{j}^{n-m}-\phi_{j-1}^{n-m}}{\Delta x}\right| \mathrm{d} x \mathrm{~d} s \\
& \leq \sum_{m=0}^{n} \sum_{j \leq j_{s}} \int_{t^{m}}^{t^{m+1}} \int_{K_{j}}\left[\left|\rho_{j}^{m}-\rho(s, x)\right| \operatorname{Lip}(\phi)+\rho_{\max }\left|\frac{\phi_{j}^{n+1-m}-\phi_{j}^{n-m}}{\Delta t}-\phi_{t}(t-s, x)\right|\right] \mathrm{d} x \mathrm{~d} s \\
& \quad+\sum_{m=0}^{n} \sum_{j \leq j_{s}} \int_{t^{m}}^{t^{m+1}} \int_{K_{j}} \operatorname{Lip}(F)\left(\left|\rho(s, x)-\rho_{j-1}^{m}\right|+\left|\rho(s, x)-\rho_{j}^{m}\right|\right) \operatorname{Lip}(\phi) \mathrm{d} x \mathrm{~d} s \\
& \quad+\sum_{m=0}^{n} \sum_{j \leq j_{s}} \int_{t^{m}}^{t^{m+1}} \int_{K_{j}} f_{\max }\left|\phi_{x}(t-s, x)-\frac{\phi_{j}^{n-m}-\phi_{j-1}^{n-m} \mid}{\Delta x}\right| \mathrm{d} x \mathrm{~d} s \underset{\Delta x \rightarrow 0}{\longrightarrow} 0,
\end{aligned}
$$

$$
\begin{aligned}
& \left|\xi^{b}(t)+\Delta x \sum_{j \leq j_{s}} \rho_{j}^{n+1} \phi_{j}^{0}\right| \leq \sum_{j \leq j_{s}} \int_{K_{j}}\left|-\rho(t, x) \phi(0, x)+\rho_{j}^{n+1} \phi_{j}^{0}\right| \mathrm{d} x \\
\leq & \sum_{j \leq j_{s}} \int_{K_{j}}\left[\left|\rho(t, x)-\rho_{j}^{n+1}\right|\|\phi\|_{\mathbf{L}^{\infty}\left(\mathbb{R}_{+} \times \mathbb{R}\right)}+\rho_{\max }\left|\phi(0, x)-\phi_{j}^{0}\right|\right] \mathrm{d} x \underset{\Delta x \rightarrow 0}{\longrightarrow} 0,
\end{aligned}
$$

and

(C)

$$
\begin{aligned}
& \left|\xi^{c}(t)-\Delta x \sum_{j \leq j_{s}} \rho_{j}^{0} \phi_{j}^{n+1}\right| \leq \sum_{j \leq j_{s}} \int_{K_{j}}\left|\rho_{0}(x) \phi(t, x)-\rho_{j}^{0} \phi_{j}^{n+1}\right| \mathrm{d} x \\
& \leq \sum_{j \leq j_{s}} \int_{K_{j}}\left[\left|\rho_{0}(x)-\rho_{j}^{0}\right|\|\phi\|_{\mathbf{L}_{\left(\mathbb{R}_{+} \times \mathbb{R}\right)}}+\rho_{\max }\left|\phi(t, x)-\phi_{j}^{n+1}\right|\right] \mathrm{d} x \underset{\Delta x \rightarrow 0}{\longrightarrow} 0 .
\end{aligned}
$$

Finally, let us prove also $\mathscr{Q}_{\Delta}^{\text {comp }}$, i.e., that $\left(\mathscr{Q}_{\Delta}\left[\rho_{\Delta}\right]\right)$ is compact in $\mathbf{L}^{\mathbf{1}}(0, T)$. 
We have that $\left(\mathscr{Q}_{\Delta}\left[\rho_{\Delta}\right]\right)$ is uniformly bounded in $\mathbf{B V}$ because

$$
\begin{aligned}
& \sum_{n=1}^{N-1}\left|\mathscr{Q}_{\Delta}\left[\rho_{\Delta}\right]\left(t^{n+1}\right)-\mathscr{Q}_{\Delta}\left[\rho_{\Delta}\right]\left(t^{n}\right)\right| \leq \operatorname{Lip}(r) \sum_{n=1}^{N-1}\left|\eta_{\Delta}\left(t^{n+1}\right)-\eta_{\Delta}\left(t^{n}\right)\right| \\
= & \operatorname{Lip}(r) \Delta t \sum_{n=1}^{N-1}\left|\sum_{m=0}^{n} \kappa^{n-m} \mathscr{F}_{j_{s}+1 / 2}^{m}-\sum_{m=0}^{n-1} \kappa^{n-1-m} \mathscr{F}_{j_{s}+1 / 2}^{m}\right| \\
= & \operatorname{Lip}(r) \Delta t \sum_{n=1}^{N-1}\left|\kappa^{0} \mathscr{F}_{j_{s}+1 / 2}^{n}+\sum_{m=0}^{n-1}\left[\kappa^{n-m}-\kappa^{n-1-m}\right] \mathscr{F}_{j_{s}+1 / 2}^{m}\right| \\
\leq & \operatorname{Lip}(r) \Delta t \sum_{n=1}^{N-1}\left[\kappa^{0} \mathscr{F}_{j_{s}+1 / 2}^{n}+\operatorname{Lip}(\kappa) \Delta t \sum_{m=0}^{n-1} \mathscr{F}_{j_{s}+1 / 2}^{m}\right] \\
\leq & 2[\kappa(0)+\operatorname{Lip}(\kappa) T] \operatorname{Lip}(r) \operatorname{Lip}(F) T \rho_{\max } .
\end{aligned}
$$

Therefore $\left(\mathscr{Q}_{\Delta}\left[\rho_{\Delta}\right]\right)$ is compact in $\mathbf{L}^{\mathbf{1}}(0, T)$.

Example 6.4. In this example we propose a discrete version of the constraint operator given in Example 4.4 Let us first rewrite the Cauchy problem 4.5] in its integral form

$$
\xi(t)=\xi_{0}\left[\rho_{0}\right]+\int_{0}^{t} H\left(\xi(s), \int_{\mathbb{R}} F(s, x, \rho(s, x)) \mathrm{d} x\right) \mathrm{d} s .
$$

Its natural discretization is then the forward Euler discretization

$$
\begin{aligned}
& \xi^{0}=\xi_{0}\left[\rho_{0}\right], \\
& \xi^{n+1}=\xi^{n}+\Delta t H\left(\xi^{n}, \Delta x \sum_{j \in \mathbb{Z}} F\left(t^{n}, x_{j}, \rho_{j}^{n}\right)\right), \\
&
\end{aligned}
$$

Assume that the sequence of approximate solutions $\left(\rho_{\Delta}\right)$ obtained with the above algorithm is bounded in $\mathbf{L}^{\infty}\left(0, T ; \mathbf{L}^{\mathbf{l}}\right)$, then $\left(\xi^{n+1}-\xi^{n}\right) / \Delta t$ is uniformly bounded by the assumptions we made on $H$. Therefore $\left(\xi_{\Delta}\right)$ is bounded uniformly in BV and we have $\mathscr{Q}_{\Delta}^{\text {comp }}$.

\section{NUMERICAL APPLICATIONS}

In this section, we first validate the numerical scheme introduced in Section 5 with an explicit solution. Then we present numerical simulations related to the constraint operators introduced in Sections 1.4.1 1.4.3. In particular, the simulations show that some drawbacks of $(1.5)$ have been removed.

7.1. Validation of the numerical scheme. We propose here to validate the numerical scheme [5.2], [5.3), [5.4] by showing its numerical convergence toward the exact solution to (1.1), [1.3), [1.4 presented in Figure 1 and obtained by an explicit analysis of the wave-front interactions, with computer-assisted computation of interaction times and front slopes. The set up for the simulation is as follows. Consider the domain of computation $[-6,0]$, take a normalized flux $f(\rho)=\rho(1-\rho)$ (namely the maximal velocity and the maximal density are assumed to be equal to one) and the weight functions $w(x)=2(1+x) \mathbf{1}_{[-1,0]}(x)$ and $\kappa(t)=2(1-t) \mathbf{1}_{[0,1]}(t)$. Assume a uniform distribution of maximal density in $\left[x_{1}, x_{2}\right]$ at time $t=0$, namely $\rho_{0}(x)=\mathbf{1}_{\left[x_{1}, x_{2}\right]}(x)$. The efficiency of the exit, $p$ is of the form

$$
p(\xi)= \begin{cases}p_{0} & \text { if } 0 \leq \xi<\xi_{1} \\ p_{1} & \text { if } \xi_{1} \leq \xi<\xi_{2} \\ p_{2} & \text { if } \xi_{2} \leq \xi \leq 1\end{cases}
$$

The explicit solution $\rho$ corresponding to the values

$$
\begin{gathered}
x_{1}=-6, \quad x_{2}=-1.2, \quad p_{0}=0.16, \quad p_{1}=66 / 625=0.1056, \quad p_{2}=24 / 625=0.0384, \\
\xi_{1}=\frac{1087}{300}-\frac{1152}{625} \sqrt{2}(3 \sqrt{3}-4)+\frac{2}{125} \log \left[\frac{2}{\sqrt{3}}\right] \sim 0.508, \quad \xi_{2} \sim 0.6911,
\end{gathered}
$$

is represented in Figure 1 .

Moreover, the time values in Figure 1 are

$$
\begin{aligned}
t_{A}=4.8, \quad t_{B}=1.2, \quad t_{C} & =2, \quad t_{D}=4, \quad t_{E} \sim 4.417, \quad t_{F}=5, \quad t_{G} \sim 5.878, \quad t_{H} \sim 12.554, \quad t_{I} \sim 105.063 \\
t_{L} & \sim 105.553, \quad t_{M} \sim 105.647, \quad t_{N} \sim 106.759, \quad t_{O} \sim 107.111, \quad t_{P} \sim 107.185, \quad t_{Q} \sim 108.464 .
\end{aligned}
$$




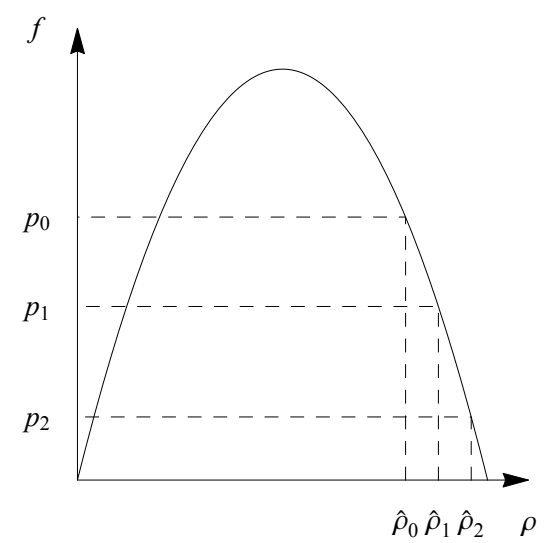

(a) The fundamental diagram.

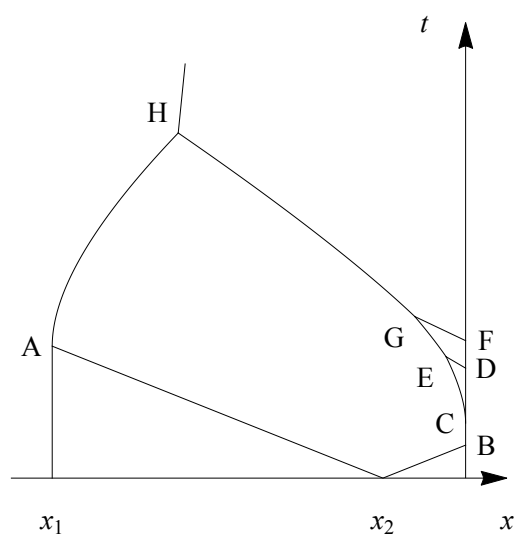

(b) The solution in the $(x, t)$-coordinates.

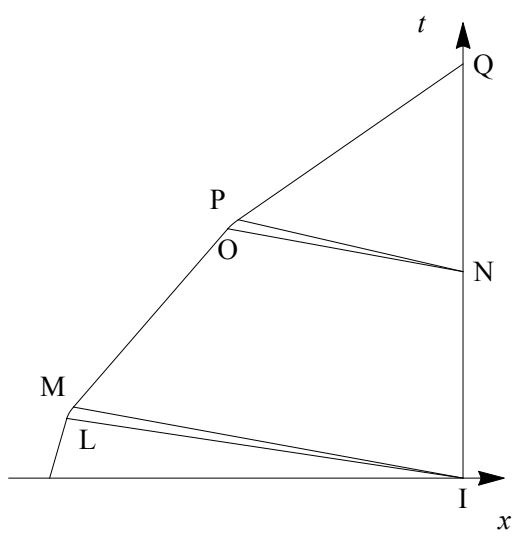

(c) The solution in the $(x, t)$-coordinates.

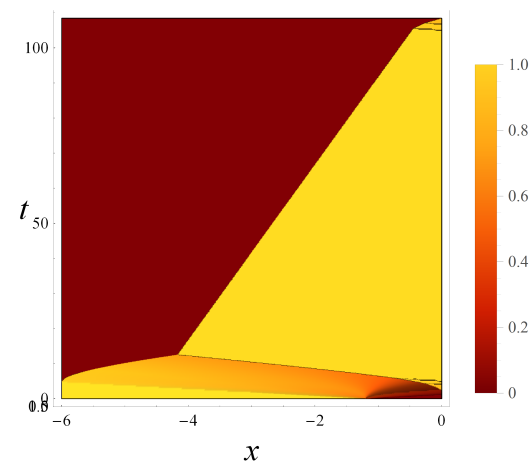

(d) The solution in the $(t, x, \rho)$-coordinates.

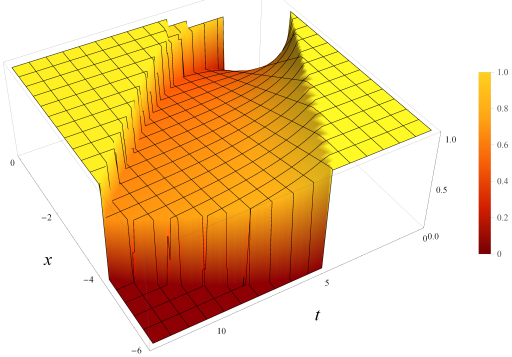

(e) The solution in the $(x, t, \rho)$-coordinates.

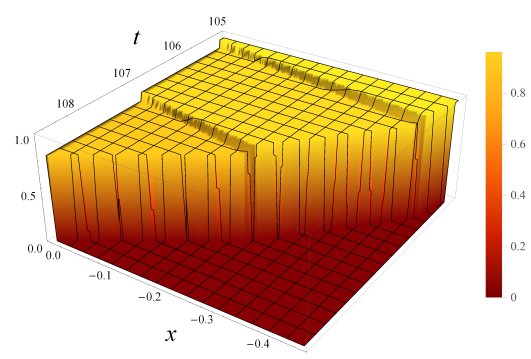

(f) The solution in the $(x, t, \rho)$-coordinates.

FIGURE 1. Representation of the solution $(t, x) \mapsto \rho(t, x)$ described in Subsection 7.1

The approximate solution is numerically computed using the Godounov numerical flux (see e.g. 34, 41), which will be used in the remaining of this paper:

$$
F(a, b)= \begin{cases}\min _{[a, b]} f & \text { if } a \leq b, \\ \max _{[b, a]} f & \text { if } a>b .\end{cases}
$$

In Figure 2] we present the numerically computed solution $x \mapsto \rho_{\Delta}(t, x)$ at different fixed times $t$ on the domain [-6, 1]. The parameters of discretization are $\Delta x=10^{-3}$ and $\Delta t=4 \times 10^{-4}$. Let us now introduce the relative $\mathbf{L}^{\mathbf{1}}$-error for the density $\rho$, at a given time $t^{n}$, defined by

$$
E_{\mathbf{L}^{1}}^{n}=\left[\sum_{j}\left|\rho\left(t^{n}, x_{j}\right)-\rho_{j}^{n}\right|\right] /\left[\sum_{j}\left|\rho\left(t^{n}, x_{j}\right)\right|\right] .
$$

In Table 1. we computed the relative $\mathbf{L}^{\mathbf{1}}$-errors for different numbers of space cells at the fixed time $t=10$ and for a fixed time step $\Delta t=10^{-4}$. As in [6, 3], we observe that the application of the constrained numerical flux [5.3] does not affect the accuracy of the scheme.

\begin{tabular}{|c|c|c|}
\hline Space step & $\mathbf{L}^{\mathbf{1}}$-error & rate of convergence \\
\hline 600 & $5.4 \times 10^{-3}$ & - \\
\hline 1200 & $2.82 \times 10^{-3}$ & 0.937 \\
\hline 2400 & $1.52 \times 10^{-3}$ & 0.915 \\
\hline 6000 & $6.8 \times 10^{-4}$ & 0.9 \\
\hline 12000 & $3.37 \times 10^{-4}$ & 0.916 \\
\hline 24000 & $1.73 \times 10^{-4}$ & 0.927 \\
\hline
\end{tabular}

TABLE 1. Relative $\mathbf{L}^{1}$-error at time $t=10$, where the constraint $\mathscr{Q}$ defined in 1.4 is used.

In Figure 3 is presented the evolution in time of the function $\xi_{\Delta}$, the approximation of

$$
\xi(t)=\int_{\mathbb{R}_{-}} \int_{0}^{t} w(x) \kappa(t-s) \rho(s, x) \mathrm{d} s \mathrm{~d} x,
$$




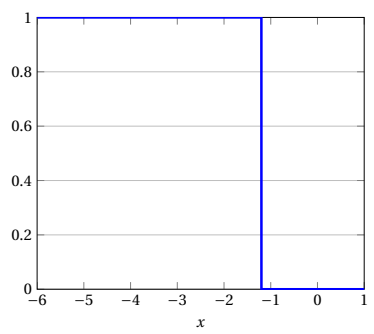

(a) $\rho_{\Delta}(0, x)$

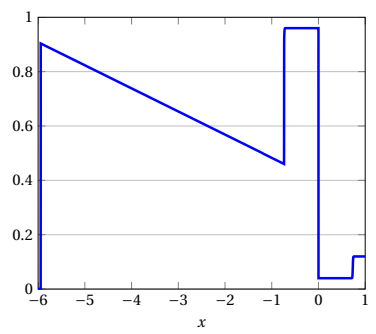

(e) $\rho_{\Delta}(5.878, x)$

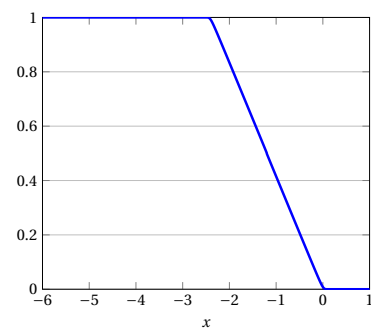

(b) $\rho_{\Delta}(1.2, x)$

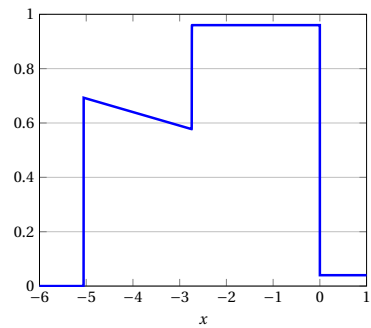

(f) $\rho_{\Delta}(10, x)$

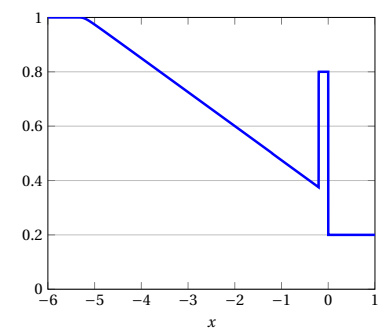

(c) $\rho_{\Delta}(4, x)$

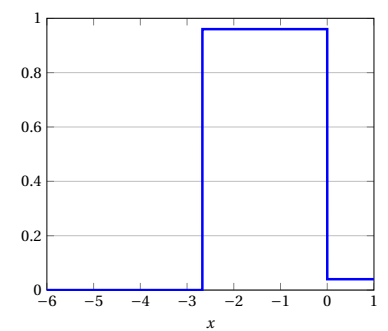

(g) $\rho_{\Delta}(50, x)$

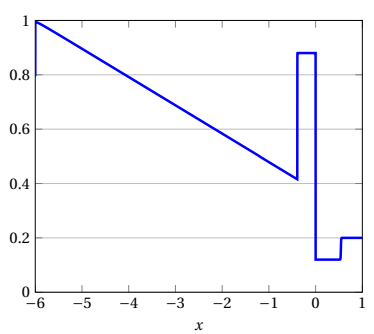

(d) $\rho_{\Delta}(4.8, x)$

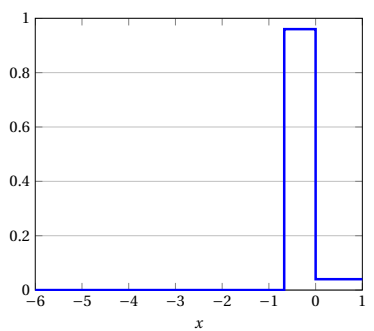

(h) $\rho_{\Delta}(100, x)$

FIGURE 2. With reference to Subsection 7.1 The numerically computed solution $x \mapsto \rho_{\Delta}(t, x)$ at different fixed times $t$.

that reports the level of "panic" in the constraint operator $\mathscr{Q}$ defined in [1.4. The main feature that can be emphasized is that the level of panic increases and disappears quickly. We shall see in Example 7.3 results when $\xi_{\Delta}$ is an approximation of $\xi$ defined by (1.8) or (1.9) that are extensions of 7.2 and that prevent the level of panic from disappearing too fast.



Figure 3. The function $t \rightarrow \xi_{\Delta}(t)$

Finally, we report in Table 2 the $\mathbf{L}^{\mathbf{1}}$-errors at $t=10$ between the exact solution and the approximate solution computed with the constraint operator $\widehat{\mathscr{Q}}$ defined in (1.4) for different numbers of space cells and for a fixed time step $\Delta t=10^{-4}$. Observe that $\mathscr{Q}$ and $\widehat{\mathscr{Q}}$ defined in 1.4 are equivalent in the sense of Definition 2.2 As expected, comparing Table 1 and Table 2 , one can easily check that the errors are essentially similar and thus the solutions computed with $\mathscr{Q}$ and $\widehat{\mathscr{Q}}$ are essentially the same.

\begin{tabular}{|c|c|c|}
\hline Space step & $\mathbf{L}^{\mathbf{1}}$-error & rate of convergence \\
\hline 600 & $6.53 \times 10^{-3}$ & - \\
\hline 1200 & $3.4 \times 10^{-3}$ & 0.941 \\
\hline 2400 & $1.83 \times 10^{-3}$ & 0.917 \\
\hline 6000 & $8.13 \times 10^{-4}$ & 0.97 \\
\hline 12000 & $4.11 \times 10^{-4}$ & 0.941 \\
\hline 24000 & $2.17 \times 10^{-4}$ & 0.93 \\
\hline
\end{tabular}

TABLE 2. Relative $\mathbf{L}^{\mathbf{1}}$-error at time $t=10$, where the constraint $\widehat{\mathscr{Q}}$ defined in 1.4 is used. 
7.2. Numerical simulations. In what follows, the exit is always located at $x=0$, we always consider the normalized flux $f(\rho)=\rho(1-\rho)$, the space weight $w(x)=2(1+x) \mathbf{1}_{[-1,0]}(x)$ and the time weight $\kappa(t)=2(1-t) \mathbf{1}_{[0,1]}(t)$. If not precised, the simulations are performed with the discretization parameters $\Delta x=10^{-3}$ and $\Delta t=4 \times 10^{-4}$.

Example 7.1. (With reference to Section 1.4.1 In this part, the set up for the simulations is the same as for the validation done in Subsection 7.1. We focus on the constraint operators $\widetilde{\mathscr{Q}}$ and $\overline{\mathscr{Q}}$ that can be interpreted as discretized versions of $\mathscr{Q}$. We take as final time $T=10$. For each of these constraint operators, we perform two types of simulations. A first type of simulations is carried out by taking a small number of discretized times and positions. We thus take the times $t_{i}=i$, for $i=0, \cdots, 10$ and positions $y_{0}=-0.8, y_{1}=-0.5, y_{2}=-0.2$ and $y_{3}=0$. We present in Figure 4 (a) and Figure 5 (a) the obtained results. For the second type of simulations, we take all the times and positions of the discretization, namely, $t_{i}=i \Delta t$, for $i=0, \cdots, 25000$ and $y_{i}=-6+i \Delta x, i=1, \cdots, 6000$. The results are depicted in Figure 4 (b) and Figure 5 (b), where we notice a good agreement with the solution presented in Figure 2(f) One can notice that, as expected, the constraint operator that corresponds to the case where data are collected by video camera is the more efficient since the two other may underestimate the importance of the congestion before the exit.

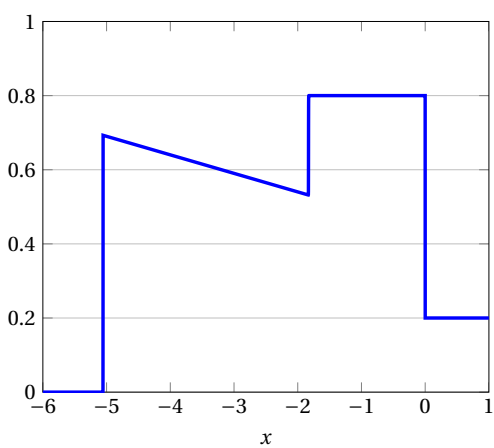

(a) $x \rightarrow \rho_{\Delta}(10, x)$ for $t_{i}=i, i=0, \cdots, 10$.



(b) $x \rightarrow \rho_{\Delta}(10, x)$ for all $t_{i}=i \Delta t, \quad i=$ $0, \cdots, 25000$.

FIgURE 4 . The solutions $x \rightarrow \rho_{\Delta}(10, x)$ corresponding to $\widetilde{\mathscr{Q}}$.

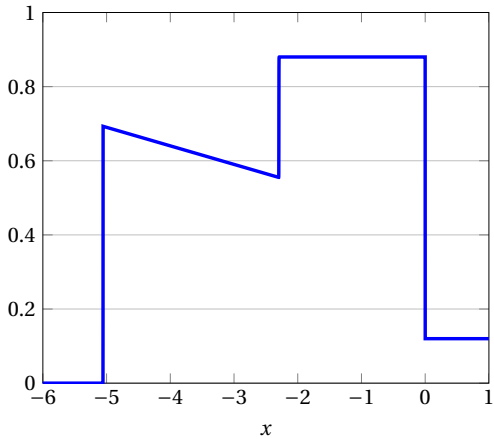

(a) $x \rightarrow \rho_{\Delta}(10, x)$ for $y_{0}=-0.8, y_{1}=-0.2$, $y_{2}=-0.5$ and $y_{3}=0$.

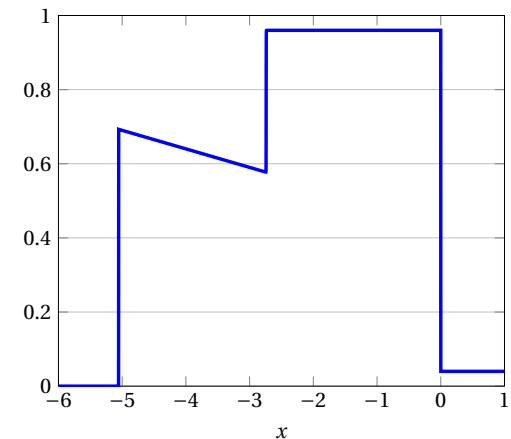

(b) $x \rightarrow \rho_{\Delta}(10, x)$ for $y_{i}=-6+i \Delta x$, $i=1, \cdots, 6000$.

Figure 5. The solutions $x \rightarrow \rho_{\Delta}(10, x)$ corresponding to $\overline{\mathscr{Q}}$.

Example 7.2. (With reference to Section 1.4.2 Here the domain of computation is $[-6,1]$ and the efficiency of the exit is the continuous function defined by

$$
p(\xi)= \begin{cases}p_{0} & \text { if } 0 \leq \xi<\xi_{1}, \\ \frac{\left(p_{0}-p_{1}\right) \xi+p_{1} \xi_{1}-p_{0} \xi_{2}}{\xi_{1}-\xi_{2}} & \text { if } \xi_{1} \leq \xi<\xi_{2}, \\ p_{1} & \text { if } \xi_{2} \leq \xi \leq 1,\end{cases}
$$

with the values

$$
p_{0}=0.21, \quad p_{1}=0.07, \quad \xi_{1}=0.35, \quad \xi_{2}=0.731 .
$$

We consider the initial density $\rho_{0}(x)=\mathbf{1}_{[-1,-0.1]}(x)$. Figure 6 shows the evolution of the fluxes at the exit computed with the constraint operators (1.5) and (1.6), where $\alpha=2$ is the maximum possible value. We can see that the value of 
the flux computed with 1.5 is immediately at the lowest level of the efficiency of the exit, whereas the one computed with [1.6] grows quickly to the highest level of the efficiency of the exit.

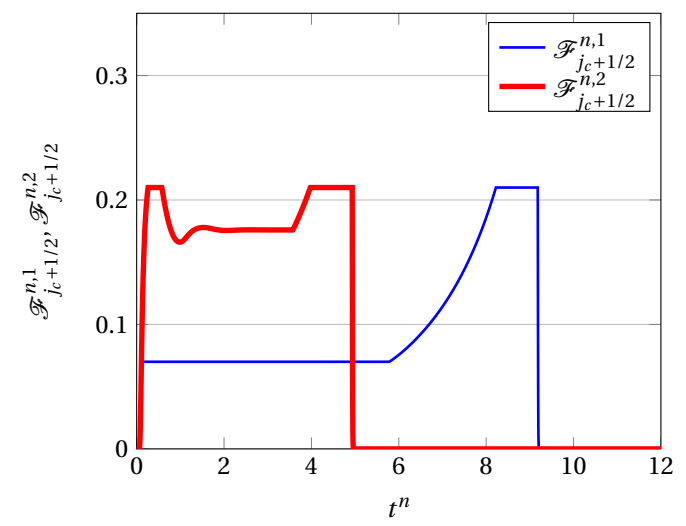

FIGURE 6. The fluxes at the exit $\mathscr{F}_{j_{c}+1 / 2}^{n, 1}$ and $\mathscr{F}_{j_{c}+1 / 2}^{n, 2}$ corresponding to the constraints [1.5 and [1.6, respectively.

Now, in order to clearly see the self-organization, we consider the domain of computation $[-4,1]$, the initial density $\rho_{0}(x)=\mathbf{1}_{[-4,-2]}(x)$ and the efficiency of the exit is still defined by 7.3 , where

$$
p_{0}=0.2, \quad p_{1}=0.1, \quad \xi_{1}=0.32, \quad \xi_{2}=0.35
$$

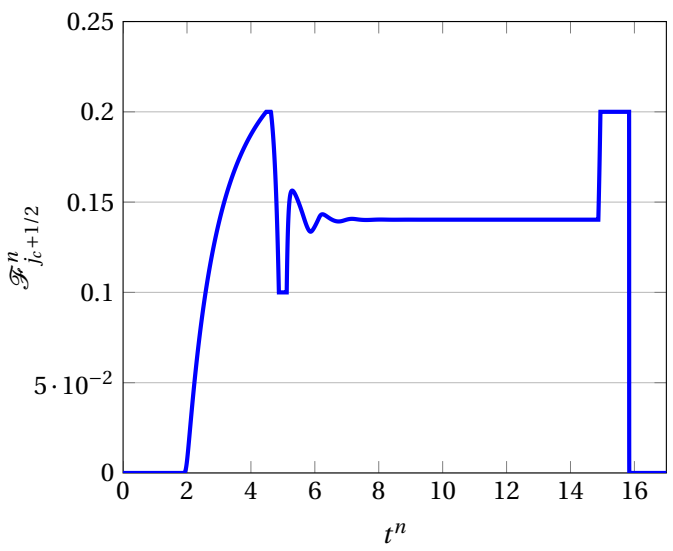

FIGURE 7. The flux at the exit corresponding to the constraint operator (1.6) showing the self-organization.

Figure 7 shows the evolution in time of the flux at the exit. We can notice that this flux first increases until it reaches the maximum level of the efficiency of the exit. Then it falls down until the lowest value of this efficiency. After a very short period it increases without reaching the maximum level of the efficiency. This is the effect of self-organization. Finally, after some time, the flux keeps a certain value until the low density observed upstream the exit allows the efficiency to recover its max value. This is in a qualitative agreement with the empirical results by Cepolina [17].

Example 7.3. (With reference to Section 1.4.3 In this part, we recall the set up introduced in [4]. The domain of computation is $[-6,1]$, the efficiency of the exit is defined by $[7.1]$ with the values

$$
p_{0}=0.21, \quad p_{1}=0.168, \quad p_{2}=0.021, \quad \xi_{1}=0.566, \quad \xi_{2}=0.731 .
$$

The initial density is $\rho_{0}(x)=\mathbf{1}_{[-5.75,-2]}(x)$. Figure 8 shows the evolution in time of $\xi_{\Delta}^{1}, \xi_{\Delta}^{2}$ and $\xi_{\Delta}^{3}$ computed using [1.5, (1.8) and [1.9] respectively for $\delta=8 \times 10^{-3}$. The graphs show that the level of panic taking into account in $\xi_{\Delta}^{2}$ or $\xi_{\Delta}^{3}$ disappears very slowly comparing to the one of $\xi_{\Delta}^{1}$.

\section{Conclusions}

In this paper, one-dimensional scalar conservation laws with nonlocal point constraints on the flux are considered. General and "easy to check" assumptions on the constraint operator are given in order to establish well-posedness of the problem using fixed point arguments. Examples of constraint operators that satisfy these assumptions are given ; they are extensions and improvements of the one considered in 4], in the sense that they remove some drawbacks 


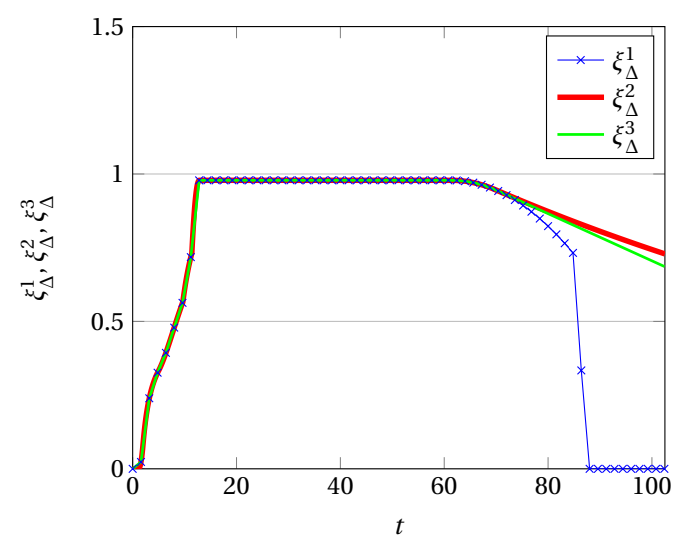

FIGURE 8. The functions $\xi_{\Delta}^{1}, \xi_{\Delta}^{2}, \xi_{\Delta}^{3}$ for [1.5], [1.8) and [1.9], respectively.

that may appear in the models based upon the latter one. A numerical scheme based on finite volume methods is developed and validated with an explicit solution. Examples of discrete constraint operators, related to the examples given in the continuous case, are given. The numerical results show that these constraints are able to reproduce features in vehicular traffic and crowd dynamics such as the self-organization. The results presented in this paper allow to consider management problems as in [20, 23].

\section{ACKNOWLEDGMENT}

The work on this paper was supported by French ANR JCJC Grant CoToCoLa (project No. ANR-11-JS01-0006) and French-Polish cooperation PHC Polonium program (project No. 331460NC). The second author also acknowledges support from the Région Franche-Comté (projet mobilité sortante a.y. 2015-16). She would like to thank the Mathematics Department of the University of Padova for the warm hospitality during the a.y. 2015-16. The third author thanks Instytut Matematyki, Uniwersytet Marii Curie-Skłodowskiej for the hospitality during the preparation of the paper. The last author thanks LMB Laboratoire de Mathématiques CNRS UMR 6623, Université de Bourgogne Franche-Comté for the hospitality during the preparation of the paper. The last author was partially supported by the INdAM-GNAMPA Project 2017 “Equazioni iperboliche con termini nonlocali: teoria e modelli”.

\section{REFERENCES}

[1] ANDREIANOv, B. The semigroup approach to conservation laws with discontinuous flux. In Hyperbolic Conservation Laws and Related Analysis with Applications, G.-Q. G. Chen, H. Holden, and K. H. Karlsen, Eds., vol. 49 of Springer Proceedings in Mathematics \& Statistics. Springer Berlin Heidelberg, 2014, pp. 1-22.

[2] Andreianov, B., Donadello, C., Razafison, U., And Rosini, M. D. Riemann problems with non-local point constraints and capacity drop. Mathematical biosciences and engineering: MBE 12, 2 (April 2015), 259-278.

[3] Andreianov, B., Donadello, C., Razafison, U., And Rosini, M. D. Qualitative behaviour and numerical approximation of solutions to conservation laws with non-local point constraints on the flux and modeling of crowd dynamics at the bottlenecks. ESAIM: M2AN50, 5 (2016), 1269-1287.

[4] Andreianov, B., Donadello, C., And Rosini, M. D. Crowd dynamics and conservation laws with nonlocal constraints and capacity drop. Mathematical Models and Methods in Applied Sciences 24, 13 (2014), 2685-2722.

[5] Andreianov, B., Donadello, C., And Rosini, M. D. A second-order model for vehicular traffics with local point constraints on the flow. Mathematical Models and Methods in Applied Sciences 26, 04 (2016), 751-802.

[6] Andreianov, B., Goatin, P., AND SEguin, N. Finite volume schemes for locally constrained conservation laws. Numerische Mathematik 115 (2010), 609-645.

[7] Andreianov, B., Karlsen, K. H., And Risebro, N. H. A theory of $L^{1}$-dissipative solvers for scalar conservation laws with discontinuous flux. Arch. Ration. Mech. Anal. 201, 1 (2011), 27-86.

[8] Andreianov, B. P., Donadello, C., Razafison, U., Rolland, J. Y., And Rosini, M. D. Solutions of the Aw-Rascle-Zhang system with point constraints. Networks and Heterogeneous Media 11, 1 (2016), 29-47.

[9] AW, A., AND RASCLE, M. Resurrection of "Second Order" Models of Traffic Flow. SIAM Journal on Applied Mathematics 60, 3 (2000), pp. 916938.

[10] Bardos, C., Leroux, A. Y., AND NédéleC, J. C. First order quasilinear equations with boundary conditions. Communications in Partial Differential Equations 4, 9 (1979), 1017-1034.

[11] Benyahia, M., AND Rosini, M. D. A macroscopic traffic model with phase transitions and local point constraints on the flow. Networks and Heterogeneous Media 12, 2 (2017), 297-317.

[12] Borsche, R., Colombo, R. M., and Garavello, M. Mixed systems:ODEs-balance laws. Journal of Differential Equations 252, 3 (2012), 2311 $-2338$.

[13] BRESSAN, A. Global solutions of systems of conservation laws by wave-front tracking. J. Math. Anal. Appl. 170, 2 (1992), 414-432.

[14] Bressan, A. Hyperbolic systems of conservation laws, vol. 20 of Oxford Lecture Series in Mathematics and its Applications. Oxford University Press, Oxford, 2000.

[15] CANCÈs, C., AND GallouËT, T. On the time continuity of entropy solutions. Journal of Evolution Equations 11, 1 (2011), 43-55.

[16] CANCÈS, C., AND SEGUIN, N. Error Estimate for Godunov Approximation of Locally Constrained Conservation Laws. SIAM Journal on Numerical Analysis 50, 6 (2012), 3036-3060. 
[17] Cepolina, E. M. Phased evacuation: An optimisation model which takes into account the capacity drop phenomenon in pedestrian flows. Fire Safety Journal 44, 4 (2009), 532 - 544.

[18] Chalons, C. Numerical approximation of a macroscopic model of pedestrian flows. SIAM Journal on Scientific Computing 29, 2 (2007), 539555.

[19] Chalons, C., Goatin, P., And Seguin, N. General constrained conservation laws. Application to pedestrian flow modeling. Networks and Heterogeneous Media 8, 2 (2013), 433-463.

[20] Colombo, R., Facchi, G., Maternini, G., And Rosini, M. On the continuum modeling of crowds. Proceedings of Hyp2008-the twelfth International Conference on Hyperbolic Problems held in the University of Maryland, College Park (2008), 517-526.

[21] Colombo, R., And Goatin, P. A well posed conservation law with a variable unilateral constraint. J. Differential Equations 234, 2 (2007), 654-675.

[22] Colombo, R. M., Goatin, P., And Rosini, M. D. A macroscopic model for pedestrian flows in panic situations. In Current advances in nonlinear analysis and related topics, vol. 32 of GAKUTO Internat. Ser. Math. Sci. Appl. Gakkōtosho, Tokyo, 2010, pp. $255-272$.

[23] Colombo, R. M., Goatin, P., And Rosini, M. D. On the modelling and management of traffic. ESAIM: Mathematical Modelling and Numerical Analysis 45, 05 (2011), 853-872.

[24] Colombo, R. M., AND Rosini, M. D. Pedestrian flows and non-classical shocks. Mathematical Methods in the Applied Sciences 28, 13 (2005), $1553-1567$.

[25] Colombo, R. M., AND Rosini, M. D. Existence of nonclassical solutions in a pedestrian flow model. Nonlinear Analysis: Real World Applications 10, 5 (2009), 2716-2728.

[26] Dafermos, C. M. Polygonal approximations of solutions of the initial value problem for a conservation law. J. Math. Anal. Appl. 38 (1972), 33-41.

[27] Dal SAnto, E., Rosini, M. D., Dymski, N., AND BENYAhiA, M. General phase transition models for vehicular traffic with point constraints on the flow. Mathematical Methods in the Applied Sciences (2017), n/a-n/a. mma.4478.

[28] Delle Monache, M., And Goatin, P. A front tracking method for a strongly coupled pde-ode system with moving density constraints in traffic flow. Discrete and Continuous Dynamical Systems - Series S 7, 3 (2014), 435-447.

[29] Delle Monache, M. L. Traffic flow modeling by conservation laws. PhD thesis, Université Nice Sophia Antipolis, 2014.

[30] Delle Monache, M. L., And Goatin, P. Scalar conservation laws with moving constraints arising in traffic flow modeling: an existence result. Journal of Differential equations 257, 11 (2014), 4015-4029.

[31] DiPerna, R. J. Global existence of solutions to nonlinear hyperbolic systems of conservation laws. J. Differential Equations 20, 1 (1976), $187-212$.

[32] Garavello, M., And Goatin, P. The Aw-Rascle traffic model with locally constrained flow. J. Math. Anal. Appl. 378, 2 (2011), 634-648

[33] Garavello, M., And Villa, S. The Cauchy problem for the Aw-Rascle-Zhang traffic model with locally constrained flow. preprint (2016).

[34] Godlewski, E., AND RaviaRT, P.-A. Numerical approximation of hyperbolic systems of conservation laws, vol. 118 of Applied Mathematical Sciences. Springer-Verlag, New York, 1996.

[35] Helbing, D., FARKAS, I., AND ViCSEK, T. Simulating dynamical features of escape panic. Nature 407, 6803 (2000), 487-490.

[36] Helbing, D., Johansson, A., And Al-Abideen, H. Z. Dynamics of crowd disasters: An empirical study. Phys. Rev. E 75 (Apr 2007 ), 046109.

[37] Holden, H., AND Risebro, N. H. Front tracking for hyperbolic conservation laws, vol. 152. Springer, 2013.

[38] Hughes, R. L. The flow of human crowds. Annual Review of Fluid Mechanics 35, 1 (2003), $169-182$.

[39] KRuZHKov, S. First order quasilinear equations with several independent variables. Mat. Sb. (N.S.) 81 (123) (1970), $228-255$.

[40] LattanZIO, C., MAURIZI, A., AND PiCCOLI, B. Moving bottlenecks in car traffic flow: A pde-ode coupled model. SIAM Journal on Mathematical Analysis 43, 1 (2011), 50-67.

[41] LeVeque, R. J. Finite volume methods for hyperbolic problems. Cambridge Texts in Applied Mathematics. Cambridge University Press, Cambridge, 2002.

[42] Lighthill, M., AND Whitham, G. On kinematic waves. II. A theory of traffic flow on long crowded roads. In Royal Society of London. Series A, Mathematical and Physical Sciences (1955), vol. 229, pp. 317-345.

[43] Lions, P.-L., Perthame, B., And TAdmor, E. A kinetic formulation of multidimensional scalar conservation laws and related equations. Journal of the American Mathematical Society 7, 1 (1994), 169-191.

[44] PANOV, E. Y. On sequences of measure-valued solutions of a first-order quasilinear equation. Matematicheskii Sbornik 185, 2 (1994), 87-106.

[45] PANOV, E. Y. Existence of strong traces for quasi-solutions of multidimensional conservation laws. J. Hyperbolic Differ. Equ. 4, 4 (2007), 729770.

[46] RichaRDS, P. I. Shock waves on the highway. Operations Research 4, 1 (1956), 42-51.

[47] Rosini, M. D. Nonclassical interactions portrait in a macroscopic pedestrian flow model. Journal of Differential Equations 246, 1 (2009), $408-427$.

[48] Rosini, M. D. The initial-boundary value problem and the constraint. Understanding Complex Systems (2013), 63-91.

[49] Sofonea, M., Avramescu, C., AND Matei, A. A fixed point result with applications in the study of viscoplastic frictionless contact problems. Communications on pure and Applied Analysis 7, 3 (2008), 645.

[50] VASSEUR, A. Strong traces for solutions of multidimensional scalar conservation laws. Arch. Ration. Mech. Anal. 160, 3 (2001), 181-193.

[51] WALK21-VI, Ed. Understanding capacity drop for designing pedestrian environments (2005).

[52] ZHANG, H. A non-equilibrium traffic model devoid of gas-like behavior. Transportation Research Part B: Methodological 36, 3 (2002), 275-290.

LMPT, CNRS UMR 7350, Université DE TOURS, PARC GRANDMONT 37200, TOURS, FranCE, E-MAIL: BORIS.ANDREIANOV@UNIV-TOURS.Fr

Laboratoire de Mathématiques CNRS UMR 6623, Univ. Bourgogne Franche-Comté, 16 route de Gray, 25030 Besançon, France, E-MAILS: \{CARLOTTA.DONADELLO / ULRICH.RAZAFISON \} @UNIV-FCOMTE.FR

Instytut Matematyki, Uniwersytet Mari Curie-SkŁodowskiej, Plac Marii Curie-SkŁodowskiej 1, 20 -031 Lublin, Poland, e-mail: MROSINI@UMCS.LUBLIN.PL 